Marquette University

e-Publications@Marquette

Electrical and Computer Engineering Faculty

Electrical and Computer Engineering, Department

Research and Publications

$11-1-2016$

\title{
Smart Power Grid Synchronization With Fault Tolerant Nonlinear Estimation
}

Xin Wang

Marquette University

Edwin E. Yaz

Marquette University, edwin.yaz@marquette.edu

Accepted version. IEEE Transactions on Power Systems, Vol. 31, No. 6 (November 2016): 4806-4816. DOI. (C) 2016 Institute of Electrical and Electronics Engineers (IEEE). Used with permission. 


\title{
e-Publications@Marquette
}

\section{Electrical and Computer Engineering Faculty Research and Publications/College of Engineering}

This paper is NOT THE PUBLISHED VERSION; but the author's final, peer-reviewed manuscript. The published version may be accessed by following the link in th citation below.

IEEE Transactions on Power Systems, Vol. 31, No. 9 (November 2016): 4806-4816. DOI. This article is (C) Institute of Electrical and Electronics Engineers (IEEE) and permission has been granted for this version to appear in e-Publications@Marquette. Institute of Electrical and Electronics Engineers (IEEE) does not grant permission for this article to be further copied/distributed or hosted elsewhere without the express permission from Institute of Electrical and Electronics Engineers (IEEE).

\section{Smart Power Grid Synchronization with Fault Tolerant Nonlinear Estimation}

\author{
Xin Wang \\ Department of Electrical and Computer Engineering, Southern Illinois University, Edwardsville, IL \\ Edwin E. Yaz \\ Department of Electrical and Computer Engineering, Marquette University, Milwaukee, WI
}

\begin{abstract}
:
Effective real-time state estimation is essential for smart grid synchronization, as electricity demand continues to grow, and renewable energy resources increase their penetration into the grid. In order to provide a more reliable state estimation technique to address the problem of bad data in the PMU-based power synchronization, this paper presents a novel nonlinear estimation framework to dynamically track frequency, voltage magnitudes and phase angles. Instead of directly analyzing in abc coordinate frame, symmetrical component transformation is employed to separate the positive, negative, and zero sequence networks. Then, Clarke's transformation is used to transform the sequence networks into the $\alpha \beta$ stationary coordinate frame, which leads to system model formulation. A novel fault tolerant extended Kalman filter based real-time
\end{abstract}


estimation framework is proposed for smart grid synchronization with noisy bad data measurements. Computer simulation studies have demonstrated that the proposed fault tolerant extended Kalman filter (FTEKF) provides more accurate voltage synchronization results than the extended Kalman filter (EKF). The proposed approach has been implemented with dSPACE DS1103 and National Instruments CompactRIO hardware platforms. Computer simulation and hardware instrumentation results have shown the potential applications of FTEKF in smart grid synchronization.

\section{SECTION I.}

\section{Introduction}

With the widespread deployment of renewable energy generations, smart load controls, energy storages, plug-in hybrid electric vehicles and other new challenges presented by the requirement of smart grid, innovative changes to our existing power infrastructures are essential. New technologies including smart meters (SMs), big data, wireless telecommunication protocols, and phasor measurement units (PMUs) are all the key elements of smart grid. This evolution will lead to stochastic operating behaviors and dynamic nature of the grid. Meanwhile, in order to address the social, economical and environmental challenges, such as the growing electricity demand and green house effect, governments have developed ambitious public policy goals. For an instance, the state of California is aiming at producing $33 \%$ of its energy from renewable energy resources by the year 2020. The number of distributed power generation systems (DPGS) rapidly increases, due to the necessity of producing more renewable and sustainable electrical energy. ${ }^{1}$ Grid synchronization with high accuracy is a critical requirement for the proper control of grid connected converters, and DPGS. Without accurate grid synchronization, our utility network may face instability or even black-out.

The purpose of this work is to develop a new real-time computing framework of power system state information, including frequency, voltage magnitude and phase angle. The positive sequence phase angle and voltage magnitude are used in energy management systems (EMS) to dynamically turn on/off the distributed energy resources in active distribution networks (ADN), and therefore to control the active and reactive power flow, and to achieve other specific operation objectives between DGs and the grid. ${ }^{2}$

A vast of power grid synchronization approaches have been reported in literature. ${ }^{3-4,5}$ These prior-art synchronization methods can be broadly categorized as follows:

a) Mathematical analysis methods are based on signal processing techniques, such as the discrete Fourier transform (DFT) or Hilbert transform (HT) analysis. Digital microprocessors are commonly used for implementing the numerical processing, thus the sampling rate is strictly required.

b) Zero-crossing method is relatively easy to achieve and design; however, it is very sensitive to grid voltage distortions such as harmonics, notches. Therefore, zero-crossing method is not very reliable in practical applications. ${ }^{6,7}$

c) Phase-Locked Loop (PLL) based synchronization techniques can implement a fast and accurate phase and frequency detection for balanced three-phase voltages. ${ }^{8-9,10,11,12,13,14,15}$ The synchronous reference frame phase-locked loop (SRF-PLL), also known as $d q$-PLL, is the most widely used method in grid connected systems. ${ }^{8}$ Based on Park's transformation, a three-phase voltage vector is transformed from $a b c$ coordinate frame to $d q$ coordinate frame. $q$-axis component contains the 
information about phase angle error and $d$-axis component is the voltage amplitude in steady state. Though SFR-PLL with low bandwidth shows good performance under balanced voltage condition, it has slow response during transient condition, and is quite sensitive to frequency fluctuation and unbalanced voltages. Several other PLL methods have been developed to improve the performance of SFR-PLL. The fixed-reference-frame PLL (FRF-PLL) proposed in ${ }^{9}$ does not require transformation of variables into the synchronous frame coordinates. In, ${ }^{10}$ an observer design is developed to enhance the PLL performance using the pole placement technique. UH-PLL proposed in ${ }^{11}$ includes a harmonic compensation mechanism to alleviate the effect of harmonic distortion, by using both of the positive and negative sequences in stationary coordinates of the fundamental and harmonic components. In, ${ }^{12}$ the second $d q$ transformation memory phase delay PLL (SMPD-PLL) is proposed to enhance the performance. Several selected major PLL synchronization methods, including $\frac{T}{4}$ delay PLL, inverse Park's transform PLL (IPT-PLL), enhanced PLL (EPLL), multiple-complex coefficient-filterbased PLL (MCCF-PLL), multiple reference frame-based PLL (MRF-PLL), second order generalized integrator-based PLL (SOGI-PLL) and multi-harmonic decoupling cell PLL (MHDC-PLL), have been implemented for grid-connected inverter systems, and compared in terms of accuracy, dynamic response, harmonic immunity, etc. in. ${ }^{13-14,15}$ However, improper modeling parameters, PLL time delays, severe unbalanced voltages, large harmonics, and the low frequency dynamic effect caused by the coupling of PLL and network impedance may result in a potential converter and generator instability issue.

d) Recent advancements on grid synchronization techniques are mostly based on the state estimation approaches, which was first proposed in. ${ }^{16}$ and 17 The weighted least square (WLS) estimation is the conventional method for static state estimation, which is discussed thoroughly in. ${ }^{18} \mathrm{~A}$ unified survey of the hierarchical WLS methods for large scale electric power system can be found in. ${ }^{19} \mathrm{In},{ }^{20} \mathrm{a}$ parallel algorithm is used, based on border virtual measurements, overlapping subsystems, and the auxiliary problem principle. Reference ${ }^{21}$ presents an application of a parallel algorithm for power system state estimation with a minimal amount of modification required to existing state estimators. In decentralized state estimation, ${ }^{22}$ the information exchange reduces to the state variables of border buses and no processing by a central coordinator is needed.

Reference ${ }^{23}$ presents a distributed method for control centers to monitor the operating condition of a power network with a Kaczmarz (row-projection) type of estimator, which exhibits finite time convergence towards the exact solution and can be used to compute WLS to a linear power system model.

Many SE methods have already been studied to incorporate the conventional supervisory control and data acquisition (SCADA) system and advanced measurement technologies such as phasor measurement units (PMU). The hierarchical scheme for distributed state estimation using synchronization phasor measurements is first introduced in. ${ }^{24}$ References ${ }^{25}$ and ${ }^{26}$ further improve this method by considering a large number of tie lines among subsystems. In, ${ }^{25}$ a two-step algorithm is proposed, which incorporates the phasor measurements and the results of the traditional phasor measurements in a post processing linear estimator, proving the same results as the nonlinear algorithm. A method for sequentially handling the conventional and PMU measurements in a two-stage procedure is proposed in. ${ }^{28}$ Reference ${ }^{29}$ presents a two-step state estimation method based on measurements provided by PMU and SCADA system. At the first step, a linear state estimator is formulated using only synchronized phasor measurements provided by PMU. At the second step, the estimated voltage phasors from the first step and the SCADA measurements are simultaneously processed by a conventional nonlinear estimator to determine the whole system state. 
In, ${ }^{30}$ and 31 different formulations of nonlinear SE methods, considering the phasor measurements and the state vectors in either rectangular or polar coordinates are discussed and compared. Free reference bus hybrid estimators are suggested in. ${ }^{32}$ Recent development involving corrupted data and poorly synchronized data in PMUs are discussed in. ${ }^{33-34,35,36,37}$

State estimation results are consistent as long as the measurement data provided to the estimation algorithm are correct. The measurements contain a certain amount of error which can be of two types, either a small statistically "well-behaved" error due to instrument inaccuracy, interference, miscalibration, etc. (disturbance and noise) or a large unpredictable error due to some sort of partial or total failure of the telemetering system, faulty signal sensing, electromagnetic interferences, system delays, transients, etc. (bad data). The error introduced by the measurement disturbance and noise is comparable with the uncertainty of most of the operational constraints (e.g., transmission line overloads), against which the results of the estimation will be checked. Therefore high filtering capacity is not a necessary requirement of the estimator. However, bad data may lead to measurements whose errors are larger than an acceptable bound compatible with the accuracy of both metering and communication systems, which can seriously distort the results of the estimation, producing completely unreliable state estimates. ${ }^{39,40}$ Some of them are easy to be identified and eliminate by using simple plausible checks, however, most bad data are not immediately detectable and are directly fed into the state estimator, which is not designed to cope with such additional errors. For the above mentioned reasons, a real time state estimator (RTSE) deployed in practical applications, must be able to detect and eliminate efficiently faulty measurements, keep good track of the state of the power system in the presence of noisy bad data measurements. Considerable research have been reported on this topic, see for instance ${ }^{41-42,43}$ and references therein.

Recent advancements in computing and phasor technologies make real-time dynamic state estimation possible with high-speed time-synchronized data provided by phasor measurement units (PMU). However, bad data in PMU measurements may greatly degrade the power quality, or even cause severe damages to the entire power systems. In order to provide more reliable dynamic state estimation addressing the problem of bad data in the PMU-based power system synchronization, ${ }^{44-45,46}$ this paper presents a novel fault tolerant extended Kalman filter for smart grid synchronization, which can provide enhanced tracking of power system state information comparing with the performances with extended Kalman filter (EKF). Although EKF may converge for state estimation, and have decent accuracy, our studies show that the proposed fault tolerant extended Kalman filter can provide more accurate prediction with smaller mean square error (MSE). In order to deal with unbalanced voltages, we apply symmetrical component transformation to separate the positive, negative, and zero sequence networks. Then, Clarke's transformation is applied to transform $a b c$ coordinate frame quantities into $\alpha \beta$ stationary coordinate frame, which leads to smart grid synchronization system model formulation. After that, the fault tolerant extended Kalman filter (FTEKF) is proposed and derived. The nonlinear estimators EKF and FTEKF are applied to estimate the frequency, voltage magnitudes and phase angles. Furthermore, computer simulations show better performance of FTEKF estimation in the presence of unbalanced voltages, bad data, and external disturbances. Last but not the least, the proposed FTEKF has been successfully implemented with dSPACE and CompactRIO hardware-in-the-loop system.

The paper is organized as follows: First, the problem formulation is investigated in Section II. Then, the state space models for the smart grid synchronization is derived in Section III. After that, Section IV presents nonlinear estimation of the extended Kalman filter and the proposed fault tolerant 
extended Kalman filter (FTEKF). In Section V, the positive sequence voltage magnitude and phase angle computation is discussed. Computer simulation results are provided in Section VIto compare the performance of EKF and FTEKF nonlinear estimation. Moreover, Section VII briefly summarizes the hardware implementation of FTEKF. Based on computer simulation studies and hardware implementation, conclusion is reached in Section VIII.

The following notation is used in this work: $v(t)$ and $v(k)$ denotes the continuous and discrete time voltage respectively. $V$ denotes the Root Mean Square (RMS) value of voltage. $\bar{V}$ is the voltage phasor. $x \in$ math calR $^{n}$ denotes $\mathrm{n}$-dimensional real vector with norm $\|x\|=\left(x^{T} x\right)^{1 / 2}$ where $(\cdot)^{T}$ indicates matrix transpose. $A \geq 0$ for a symmetric matrix denotes a positive semi-definite matrix. $P$ denotes the covariance matrix. $\bar{x}$ is the mean value for $x . \operatorname{Prob}(\cdot)$ is the probability of an event. $E(x)=\bar{x}$ is the mean/expectation value of a random variable $x . x \sim(\bar{x}, X)$ denotes a random variable $x$ with arbitrary distribution with mean $\bar{x}$ and covariance $X . \delta_{k-j}$ is the Kronecker delta function; that is, $\delta_{k-j}=$ 1 when $k=j$; and $\delta_{k-j}=0$ when $k \neq j$. Let $A$ and $B$ be $n \times m$ matrices, the Hadamard product of $A$ and $B$ is denoted by $A \otimes B$, and is defined as $[A \otimes B]_{i, j}=[A]_{i j}[B]_{i j}$ for $1 \leq i \leq n, 1 \leq j \leq m$. Matrix form of Rayleigh's inequality is also used in the derivation of this work, which can be stated as: for $X=X^{T} \in$ mathcalR $^{n \times n}$ and $Y \in$ mathcalR ${ }^{m \times n}$, the matrix inequality $\lambda_{\min }(X) Y Y^{T} \leq Y X Y^{T} \leq$ $\lambda_{\max }(X) Y Y^{T}$ holds.

\section{SECTION II.}

\section{Problem Formulation}

The general form of three-phase electrical power grid voltages can be expressed as 49,50

$$
\begin{aligned}
& v_{a}(t)=\sqrt{2} V_{a} \cos \left(\omega t+\phi_{a}\right) \\
& v_{b}(t)=\sqrt{2} V_{b} \cos \left(\omega t+\phi_{b}\right)(1) \\
& v_{c}(t)=\sqrt{2} V_{c} \cos \left(\omega t+\phi_{c}\right)
\end{aligned}
$$

where $v_{a}(t), v_{b}(t), v_{c}(t)$ are the instantaneous unbalanced three phase voltages; $t$ is time in seconds; $\omega$ is electrical angular frequency in rad/s. $V i$ and $\phi_{i}(i=a, b, c)$ are the corresponding root mean square (RMS) voltage amplitudes and phase angles. It is worthwhile mentioning that the three phase voltages are not necessarily balanced, so they may not have the same magnitude, nor the phase angle difference of $120^{\circ}$.

The discrete-time three phase voltages with external disturbances and noises can be obtained from (1) as

$$
\begin{aligned}
& v_{a}(k)=\sqrt{2} V_{a} \cos \left(\omega k T+\phi_{a}\right) \\
& v_{b}(k)=\sqrt{2} V_{b} \cos \left(\omega k T+\phi_{b}\right)(2) \\
& v_{c}(k)=\sqrt{2} V_{c} \cos \left(\omega k T+\phi_{c}\right)
\end{aligned}
$$


where $k$ is the sampling instant $k=0,1,2,3, \ldots$. For sampling period $T, x(k)=x(k T)$ equals the magnitude of $x(t)$ at the $k$ th sampling instant. The grid frequency and sampling frequency are considered to be $60 \mathrm{~Hz}, 2400 \mathrm{~Hz}$, respectively in our case.

Denote $v(k)=\left[v_{a}(k), v_{b}(k), v_{c}(k)\right]^{T}$ as the three-phase voltage vector. According to symmetrical component transformation (Fortescue's transformation), three phase voltages can be expressed in term of positive, negative, and zero sequence voltages 47,48

$$
v(k)=v_{0}(k)+v_{p}(k)+v_{n}(k)
$$

where $v(k)$ represents the instantaneous three phase voltages, and $v_{i}(i=0, p, n)$ denote the zero, positive, and negative sequence voltages

$$
\begin{aligned}
& v_{p}(k)=\sqrt{2} V_{p}\left[\cos \left(\theta_{p}\right), \cos \left(\theta_{p}-120^{\circ}\right), \cos \left(\theta_{p}+120^{\circ}\right)\right]^{T} \\
& v_{n}(k)=\sqrt{2} V_{n}\left[\cos \left(\theta_{n}\right), \cos \left(\theta_{n}+120^{\circ}\right), \cos \left(\theta_{n}-120^{\circ}\right)\right]^{T}(4) \\
& v_{0}(k)=\sqrt{2} V_{0}\left[\cos \left(\theta_{0}\right), \cos \left(\theta_{0}\right), \cos \left(\theta_{0}\right)\right]^{T}
\end{aligned}
$$

where $\theta_{i}=\theta_{i}(k)=\omega k T+\phi_{i}$ for $i=(p, n, 0)$, which are the positive, negative and zero sequence phase angles, respectively. A fast and precise detection of the positive sequence of grid voltage is a critical step of controlling the connection of DGs to power grid.

Based on symmetrical component transformation, the $a b c$ coordinate frame three-phase voltage phasors can be separated to the positive, negative, and zero sequence phasors.

$$
\left[\begin{array}{l}
\bar{V}_{a} \\
\bar{V}_{b} \\
\bar{V}_{c}
\end{array}\right]=\left[\begin{array}{ccc}
1 & 1 & 1 \\
1 & a^{2} & a \\
1 & a & a^{2}
\end{array}\right]\left[\begin{array}{l}
\bar{V}_{0} \\
\bar{V}_{p} \\
\bar{V}_{n}
\end{array}\right]
$$

where $\bar{V}_{i}$ for $i=(0, p, n)$ are zero, positive, and negative sequence voltage phasors, and $a=1 \angle 120^{\circ}$. Applying Clarke's transformation, we can transform abc coordinate frame voltage phasors to stationary $\alpha \beta$ coordinate frame voltage phasors.

$$
\left[\begin{array}{l}
\bar{V}_{\alpha} \\
\bar{V}_{\beta}
\end{array}\right]=\frac{2}{3}\left[\begin{array}{ccc}
1 & -\frac{1}{2} & -\frac{1}{2} \\
0 & \frac{\sqrt{3}}{2} & -\frac{\sqrt{3}}{2}
\end{array}\right]\left[\begin{array}{l}
\bar{V}_{a} \\
\bar{V}_{b} \\
\bar{V}_{c}
\end{array}\right] \text { 6) }
$$

Using (5) and (6), we get 


$$
\left[\begin{array}{c}
\bar{V}_{\alpha} \\
\bar{V}_{\beta}
\end{array}\right]=\left[\begin{array}{cc}
1 & 1 \\
-j & j
\end{array}\right]\left[\begin{array}{c}
\bar{V}_{p} \\
\bar{V}_{n}
\end{array}\right]
$$

Voltage phasors $(7)$ can be expressed as discrete-time instantaneous voltages $v_{\alpha}(k)$ and $v_{\beta}(k)$ as follows

$$
\begin{aligned}
v_{\alpha}(k) & =\sqrt{2} V_{p} \cos \left(\omega k T+\phi_{p}\right)+\sqrt{2} V_{n} \cos \left(\omega k T+\phi_{n}\right) \\
& =\sqrt{2}\left(V_{p} \cos \phi_{p}+V_{n} \cos \phi_{n}\right) \cos \omega k T- \\
& \sqrt{2}\left(V_{p} \sin \phi_{p}+V_{n} \sin \phi_{n}\right) \sin \omega k T \\
& =\sqrt{2} V_{\alpha} \cos \left(\omega k T+\phi_{\alpha}\right) \\
v_{\beta}(k) & =\sqrt{2} V_{p} \sin \left(\omega k T+\phi_{p}\right)-\sqrt{2} V_{n} \sin \left(\omega k T+\phi_{n}\right) \\
& =\sqrt{2}\left(V_{p} \cos \phi_{p}-V_{n} \cos \phi_{n}\right) \sin \omega k T+ \\
& \sqrt{2}\left(V_{p} \sin \phi_{p}-V_{n} \sin \phi_{n}\right) \cos \omega k T \\
& =\sqrt{2} V_{\alpha} \cos \left(\omega k T+\phi_{\beta}\right)
\end{aligned}
$$

Note that zero sequence quantities in (7) are zeros after applying Clarke's transformation.

\section{SECTION III.}

State Space System Dynamics

Based on (8) and (9), and applying the discretization process with sampling period $T$, the state space variables of grid voltage synchronization are chosen as follows

$$
\begin{aligned}
& x_{1}(k)=\sqrt{2} V_{\alpha} \cos \left(k \omega T+\phi_{\alpha}\right) \\
& x_{2}(k)=\sqrt{2} V_{\alpha} \sin \left(k \omega T+\phi_{\alpha}\right) \\
& x_{3}(k)=\sqrt{2} V_{\beta} \cos \left(k \omega T+\phi_{\beta}\right)(10) \\
& x_{4}(k)=\sqrt{2} V_{\beta} \sin \left(k \omega T+\phi_{\beta}\right) \\
& x_{5}(k)=\omega
\end{aligned}
$$

Denote $t=k T$ and $T=1 / f_{S}$, where $T$ is sampling time and $f_{s}$ is sampling frequency. From (10), we formulate the system model as follows 


$$
\begin{aligned}
& x_{1}(k+1)=x_{1}(k) \cos \left(x_{5}(k)\right)-x_{2}(k) \sin \left(x_{5}(k)\right) \\
& x_{2}(k+1)=x_{1}(k) \sin \left(x_{5}(k)\right)+x_{2}(k) \cos \left(x_{5}(k)\right) \\
& x_{3}(k+1)=x_{3}(k) \cos \left(x_{5}(k)\right)-x_{4}(k) \sin \left(x_{5}(k)\right)(11) \\
& x_{4}(k+1)=x_{3}(k) \sin \left(x_{5}(k)\right)+x_{4}(k) \cos \left(x_{5}(k)\right) \\
& x_{5}(k+1)=x_{5}(k)
\end{aligned}
$$

Note that the system/process noise is assumed to be zero in (11). The measurement equations are defined as

$$
\begin{aligned}
& y_{1}(k)=x_{1}(k)+w_{1}(k) \\
& y_{2}(k)=x_{3}(k)+w_{2}(k)
\end{aligned}
$$

where $w_{1}(k)$ and $w_{2}(k)$ are considered to be external disturbances.

\section{SECTION IV.}

Computation of the Positive Sequence Voltage Magnitude and Phase Angle

Taking matrix inverse of (7), we have

$$
\left[\begin{array}{l}
\bar{V}_{p} \\
\bar{V}_{n}
\end{array}\right]=\frac{1}{2}\left[\begin{array}{cc}
1 & j \\
1 & -j
\end{array}\right]\left[\begin{array}{c}
\bar{V}_{\alpha} \\
\bar{V}_{\beta}
\end{array}\right]
$$

Expanding the first row of the phasor matrix $\underline{(13)}$ and applying Euler's Identity, we get the positive voltage phasor

$$
\begin{aligned}
\bar{V}_{p} & =V_{p} \angle \theta_{p}=\frac{1}{2}\left(\bar{V}_{\alpha}+j \bar{V}_{\beta}\right) \\
& =0.5\left[\left(V_{\alpha} \cos \theta_{\alpha}-V_{\beta} \sin \theta_{\beta}\right)+j\left(V_{\alpha} \sin \theta_{\alpha}+V_{\beta} \cos \theta_{\beta}\right)\right]
\end{aligned}
$$

Therefore, the positive sequence voltage magnitude and phase angle can be obtained as follows ${ }^{49}$

$$
\begin{aligned}
& \theta_{p}=\tan ^{-1} \frac{V_{\alpha} \sin \left(\theta_{\alpha}\right)+V_{\beta} \cos \left(\theta_{\beta}\right)}{V_{\alpha} \cos \left(\theta_{\alpha}\right)-V_{\beta} \sin \left(\theta_{\beta}\right)} \\
& V_{p}=\frac{1}{2} \sqrt{\left(V_{\alpha} \sin \theta_{\alpha}+V_{\beta} \cos \theta_{\beta}\right)^{2}+\left(V_{\alpha} \cos \theta_{\alpha}-V_{\beta} \sin \theta_{\beta}\right)^{2}}(15)
\end{aligned}
$$

At every time step $k$, based on the state estimate, we have the estimated positive sequence voltage magnitude and phase angle 


$$
\begin{gathered}
\hat{\theta_{p}}(k)=\tan ^{-1} \frac{\hat{x}_{2}(k)+\hat{x}_{3}(k)}{\hat{x}_{1}(k)-\hat{x}_{4}(k)} \\
\hat{V_{p}}(k)=\frac{1}{2} \sqrt{\left(\hat{x}_{2}(k)+\hat{x}_{3}(k)\right)^{2}+\left(\hat{x}_{1}(k)-\hat{x}_{4}(k)\right)^{2}}
\end{gathered}
$$

\section{SECTION V.}

\section{Nonlinear Estimation}

A. Extended Kalman Filter

Consider the discrete time nonlinear power system dynamics and measurement equation given as follows

$$
\begin{gathered}
x_{k+1}=f\left(x_{k}, u_{k}, v_{k}\right) \\
y_{k}=h\left(x_{k}, u_{k}, w_{k}\right)
\end{gathered}
$$

The extended Kalman filter is applied to estimate the frequency, voltage amplitudes and phase angles. EKF state estimation consists two steps: time update and measurement update. ${ }^{51,52}$ Define the following Jacobian matrices

$$
\begin{aligned}
& A_{k}=\left.\frac{\partial f}{\partial x}\right|_{x=\hat{x}_{k}}, F_{k}=\left.\frac{\partial f}{\partial v}\right|_{x=\hat{x}_{k}} \\
& C_{k}=\left.\frac{\partial h}{\partial x}\right|_{x=\hat{x}_{k}}, G_{k}=\left.\frac{\partial h}{\partial w}\right|_{x=\hat{x}_{k}}
\end{aligned}
$$

For time update, we need to compute the priori covariance and priori state estimate

$$
\begin{gathered}
P_{k}^{-}=A_{k-1} P_{k-1}^{+} A_{k-1}^{T}+F_{k-1} V_{k-1} F_{k-1}^{T} \\
\hat{x}_{k}^{-} \\
=f_{k-1}\left(\hat{x}_{k-1}^{+}, u_{k-1}, 0\right)
\end{gathered}
$$

where $V_{k}$ is the covariance matrix of process noise at time step $k$. We neglect the process noise, so the second term in (21) is zero. $\hat{x}^{-}$is the priori state estimate and $P_{k}^{-}$is priori covariance matrix. Based on the grid synchronization model (11), $A$ can be defined by a $5 \times 5$ matrix as

$$
A=\left(\begin{array}{ccccc}
\cos x_{5} & -\sin x_{5} & 0 & 0 & A_{15} \\
\sin x_{5} & \cos x_{5} & 0 & 0 & A_{25} \\
0 & 0 & \cos x_{5} & -\sin x_{5} & A_{35} \\
0 & 0 & \sin x_{5} & \cos x_{5} & A_{45} \\
0 & 0 & 0 & 0 & 1
\end{array}\right)
$$


where

$$
\begin{aligned}
& A_{15}=-x_{1} \sin x_{5}-x_{2} \cos x_{5} \\
& A_{25}=x_{1} \cos x_{5}-x_{2} \sin x_{5} \\
& A_{35}=-x_{3} \sin x_{5}-x_{4} \cos x_{5} \\
& A_{45}=x_{3} \cos x_{5}-x_{4} \sin x_{5}
\end{aligned}
$$

The measurement update can be summarized as

$$
\begin{aligned}
& K_{k}=P_{k}^{-} C_{k}^{T}\left(C_{k} P_{k}^{-} C_{k}^{T}+G_{k} W_{k} G_{k}^{T}\right)^{-1} \\
& \hat{x}_{k}^{+}=\hat{x}_{k}^{-}+K_{k}\left[y_{k}-h_{k}\left(\hat{x}^{-}, 0\right)\right] \\
& P_{k}^{+}=\left(I-K_{k} C_{k}\right) P_{k}^{-}
\end{aligned}
$$

where $\hat{x}_{k}^{+}$is posteriori state estimate; $P_{k}^{+}$is posteriori covariance matrix. Based on the measurement equation $\underline{(12)}, C_{k}$ can be computed as

$$
C_{k}=\left(\begin{array}{lllll}
1 & 0 & 0 & 0 & 0 \\
0 & 0 & 1 & 0 & 0
\end{array}\right)(27)
$$

B. Fault Tolerant Extended Kalman Filter

In order to provide a more reliable and effective state estimation of power systems against various bad data, disturbances and noises, we propose the locally unbiased, resilient, minimum variance state estimator, the fault tolerant extended Kalman filter (FTEKF), for nonlinear power system process and measurement equations (11) and (12). It means that the state estimator is unbiased for small error values, has robustness against gain perturbations, and achieves minimum value for an upper bound on the estimation error covariance.

Consider the discrete-time nonlinear stochastic power system process and measurement equations as follows

$$
\begin{gathered}
x_{k+1}=f\left(x_{k}\right)+v_{k} \\
y_{k}=\left(\begin{array}{c}
y_{k}^{1} \\
y_{k}^{2} \\
\vdots \\
y_{k}^{p}
\end{array}\right)=\left(\begin{array}{c}
\gamma_{k}^{1} h^{1}\left(x_{k}\right)+w_{k}^{1} \\
\gamma_{k}^{2} h^{2}\left(x_{k}\right)+w_{k}^{2} \\
\vdots \\
\gamma_{k}^{p} h^{p}\left(x_{k}\right)+w_{k}^{p}
\end{array}\right)
\end{gathered}
$$

where

$x_{k} \in$ mathcalR $^{n}$ state vector 
$v_{k} \in$ mathcalR $^{n}$ system noise

$y_{k} \in$ mathcalR $^{p}$ measurement vector

$w_{k}^{i} \in$ mathcalR measurement noise in each phasor measurement unit and $\mathrm{Wk}=[\mathrm{W} 1 \mathrm{k}, \mathrm{W} 2 \mathrm{k}, \ldots, \mathrm{Wpk}] \mathrm{T}$ $f, h \quad$ differentiable non-linear vector functions

The mean of initial state $x_{0}$ is $E\left[x_{0}\right]=\bar{x}_{0}$ and covariance of initial state $x_{0}$ is $X_{0}=E\left[\left(x_{0}-\bar{x}_{0}\right)\left(x_{0}-\right.\right.$ $\left.\bar{x}_{0}\right)^{T}$. The noise processes, $v_{k}$ and $w_{k}$, are white, zero mean, uncorrelated with each other and with $x_{0}$, and have covariance $V_{k}$ and $W_{k}$, respectively.

$$
\begin{gathered}
v_{k} \sim\left(0, V_{k}\right), w_{k} \sim\left(0, W_{k}\right), \\
E\left[v_{k} v_{j}^{T}\right]=V_{k} \delta_{k-j}, E\left[w_{k} w_{j}^{T}\right]=W_{k} \delta_{k-j}, \\
E\left[v_{k} w_{j}^{T}\right]=0, E\left[v_{k} x_{0}^{T}\right]=0, E\left[w_{k} x_{0}^{T}\right]=0
\end{gathered}
$$

The scalar binary Bernoulli distributed random variables $\gamma_{k}^{i}$ are with mean $\pi_{i}$ and variance $\pi_{i}(1-$ $\pi_{i}$ ) whose possible outcomes 0, 1 are defined as $\operatorname{Prob}\left(\gamma_{k}^{i}=1\right)=\pi_{i}$ and $\operatorname{Prob}\left(\gamma_{k}^{i}=0\right)=1-\pi_{i}$. The formulation involves hard measurement failures, where the PMU either works properly or bad data occurs.

Denote the measurement vector of $p$ phasor measurement units at $y(k)=\left[y^{1}(k), y^{2}(k), \ldots, y^{p}(k)\right]^{T}$, and the measurement estimate vector at $t=k T$ as $\hat{y}(k)=\left[\hat{y}^{1}(k), \hat{y}^{2}(k), \ldots, \hat{y}^{p}(k)\right]^{T}$. Let $r^{i}(k)$ be the $i$ th component of residual vector defined by the difference between the ith PMU measurement $y^{i}(k)$ and the corresponding estimated measurement $\hat{y}^{i}(k)$.

$$
r(k)=y(k)-\hat{y}(k) \cong C_{k}[x(k)-\hat{x}(k)]+w(k)(30)
$$

It is known that $r^{i}(k)$ is approximately a white Gaussian process with zero mean and covariance matrix $\sigma_{r}^{2}(k)$ given by

$$
\sigma_{r}^{2}(k)=C_{k} P_{k} C_{k}^{T}+W_{k}(31)
$$

where $C_{k} P_{k} C_{k}^{T}$ is the error covariance of $C_{k}[x(k)-\hat{x}(k)]$, and $W_{k}$ is the measurement noise covariance of $w(k)$. The sets of received and estimated measurements are mutually independent.

Define the normalized residual vector $r_{N}(k)$, at time step $k . \sigma_{r}^{i}(k)$ is the ith diagonal element of standard deviation matrix $\sqrt{\sigma_{r}^{2}(k)}$. The components of vector $r$ are normalized and submitted to the following statistical validation:

$$
r_{N}^{i}(k)=\frac{r^{i}(k)}{\sigma_{r}^{i}(k)}(32)
$$


If $\left|r_{N}^{i}(k)\right| \leq$ mathcalB and the measurement is compatible with the accuracy of both metering and communication systems, we assume that the ith PMU works properly at time step $k$, i.e., $\gamma_{k}^{i}=1$.

If $\left|r_{N}^{i}(k)\right|>$ mathcalB, or the measurement is not compatible with the accuracy of both metering and communication systems, we consider that bad data occurs at the ith PMU, i.e., $\gamma_{k}^{i}=0$. The threshold mathcalB is determined by the test confidence, i.e., the detection threshold for the test can be set to 3 with a $99.72 \%$ confidence level. ${ }^{38}$ Note that the detection threshold value determines the test confidence level. If the detection threshold adopted for normalized residual test is 2 , then the confidence level is $94.46 \%$. If the detection threshold is 2.5 , then the confidence level is $98.76 \%$. Typical bad data measurement detection threshold mathcalB is set to 3 , which is used for our application. Therefore, if the normalized residue exceeds 3 , then the corresponding measurement can be flagged for elimination. A simplified pseudo-algorithm that summarizes this process is provided in Algorithm 1.

Algorithm 1 Bad Data Detection

Require: acceptable threshold mathcalB

1. Procedure Bad Data Detection

2. for every sampling time step $k$ do

3. Obtain measurements $y(k)=\left[y^{1}(k), \ldots ., y^{p}(k)\right]^{T}$

4. at time $t=k T$, for each PMU index $i(i=1 \rightarrow p)$

5. Compute $r(k)=y(k)-\hat{y}(k)$

6. Compute standard deviation of residual $\sigma_{r}(k)$

7. Compute the normalized residual $r_{N}(k)=\frac{r(k)}{\sigma_{r}(k)}$

8. if $\left|r_{N}^{i}(k)\right| \leq$ mathcalBthen,

9. $y^{i}(k)=$ dynamic d ata,

10. Assume $\gamma_{k}^{i}=1$

11. end if

12. if $\left|r_{N}^{i}(k)\right|>$ mathcalB then,

13. $y^{i}(k)=$ bad $\mathrm{d}$

ata,

14. Assume $\gamma_{k}^{i}=0$

15. end if

16. end for

17. end procedure

By denoting

$$
\begin{aligned}
& \Gamma_{k}=\operatorname{diag}\left[\gamma_{k}^{1}, \gamma_{k}^{2}, \ldots, \gamma_{k}^{p}\right] \\
& h\left(x_{k}\right)=\operatorname{diag}\left[h^{1}\left(x_{k}\right), h^{2}\left(x_{k}\right), \ldots, h^{p}\left(x_{k}\right)\right]
\end{aligned}
$$


the measurement equation can be written as

$$
y_{k}=\Gamma_{k} h\left(x_{k}\right)+w_{k}(35)
$$

Our goal is to estimate the state vector $x_{k}$ based on our knowledge of system dynamics and the availability of the noisy measurement $y_{k}$ under the effect of sensor failures. The following discrete time nonlinear Luenberger observer is considered in this work.

$$
\hat{x}_{k+1}=f\left(\hat{x}_{k}\right)+\left(K_{k}+\Delta_{k}\right)\left(y_{k}-\bar{\Gamma}_{k} h\left(\hat{x}_{k}\right)\right)
$$

Although the filter gain should be $K_{k}$, due to computational or tuning uncertainties, it is erroneously implemented as $K_{k}+\Delta_{k}$. The term $\bar{\Gamma}_{k}$ is defined as

$$
\bar{\Gamma}_{k}=E\left[\Gamma_{k}\right]=\operatorname{diag}\left[\pi_{1}, \pi_{2}, \ldots, \pi_{p}\right]
$$

$K_{k}$ is the feedback gain with additive uncertainty $\Delta_{k}$. The uncertainty $\Delta_{k}$, is assumed to have zero mean, bounded second moment and be uncorrelated with initial state, process and measurement noises, i.e.,

$$
E\left[\Delta_{k} \Delta_{k}^{T}\right] \leq \delta I, E\left[\Delta_{k}^{T} x_{0}\right]=0, E\left[\Delta_{k}^{T} v_{k}\right]=0, E\left[\Delta_{k} w_{k}\right]=0
$$

Theorem 1

The fault tolerant extended Kalman filter is defined as follows:

1. Initialization

$$
\begin{gathered}
\hat{x}_{0}=E\left[x_{0}\right] \\
P_{0}=E\left[\left(x_{0}-\hat{x}_{0}\right)\left(x_{0}-\hat{x}_{0}\right)^{T}\right]
\end{gathered}
$$

2. Computation of Jacobian matrices

$$
A_{k}=\left.\frac{\partial f}{\partial x}\right|_{x=\hat{x}_{k}}, C_{k}=\left.\frac{\partial h}{\partial x}\right|_{x=\hat{x}_{k}}(40)
$$

3. For time steps $k=1,2,3, \ldots$, the estimator propagates by calculating the feedback gain

$$
\begin{aligned}
K_{k}^{o}= & \left(A_{k} P_{k} C_{k}^{T} \bar{\Gamma}_{k}^{T}\right)\left[\bar{\Gamma}_{k} C_{k} P_{k} C_{k}^{T} \bar{\Gamma}_{k}^{T}+\right. \\
& \left.\Upsilon \otimes\left(h\left(\hat{x}_{k}\right) h^{T}\left(\hat{x}_{k}\right)+C_{k} P_{k} C_{k}^{T}\right)+W_{k}\right]^{-1}
\end{aligned}
$$

from an upper bound on the local estimation error co variance 


$$
\begin{aligned}
& P_{k+1}=A_{k} P_{k} A_{k}^{T}+V_{k}+\lambda_{\max }\left\{\bar{\Gamma}_{k} C_{k} P_{k} C_{k}^{T} \bar{\Gamma}_{k}^{T}+W_{k}+\right. \\
& \left.\Upsilon \otimes\left(h\left(\hat{x}_{k}\right) h^{T}\left(\hat{x}_{k}\right)+C_{k} P_{k} C_{k}^{T}\right)\right\} \delta I \\
& -\left(A_{k} P_{k} C_{k}^{T} \bar{\Gamma}_{k}^{T}\right)\left[\bar{\Gamma}_{k} C_{k} P_{k} C_{k}^{T} \bar{\Gamma}_{k}^{T}+\right. \\
& \left.\Upsilon \otimes\left(h\left(\hat{x}_{k}\right) h^{T}\left(\hat{x}_{k}\right)+C_{k} P_{k} C_{k}^{T}\right)+W_{k}\right]^{-1}\left(\bar{\Gamma}_{k} C_{k} P_{k} A_{k}^{T}\right)
\end{aligned}
$$

to be used in updating the state estimate as

$$
\hat{x}_{k+1}=f\left(\hat{x}_{k}\right)+\left(K_{k}^{o}+\Delta_{k}\right)\left(y_{k}-\bar{\Gamma}_{k} h\left(\hat{x}_{k}\right)\right)
$$

where

$$
\begin{array}{r}
\Upsilon=\operatorname{diag}\left[\pi_{1}\left(1-\pi_{1}\right), \pi_{2}\left(1-\pi_{2}\right), \ldots, \pi_{p}\left(1-\pi_{p}\right)\right] \\
=\left(\begin{array}{cccc}
\pi_{1}\left(1-\pi_{1}\right) & 0 & \cdots & 0 \\
0 & \ddots & \ddots & \vdots \\
\vdots & \ddots & \ddots & 0 \\
0 & \cdots & 0 & \pi_{p}\left(1-\pi_{p}\right)
\end{array}\right)
\end{array}
$$

\section{Proof}

The proof of this theorem can be found in..$^{53}$ Note that the upper bound covariance in (42) is obtained based on Rayleigh's inequality. It can shown that EKF is a special case of the proposed FTEKF, when there are no measurement failures.

\section{Remark 1}

As a limiting case, if we have no perturbations on the estimator gain, i.e., $\delta=0$, then the following estimator can be derived following a similar procedure to the previously given. In this case, the robust optimal feedback gain is

$$
\begin{aligned}
K_{k}^{o}= & \left(A P_{k} C_{k}^{T} \bar{\Gamma}_{k}^{T}\right)\left[\bar{\Gamma}_{k} C_{k} P_{k} C_{k}^{T} \bar{\Gamma}_{k}^{T}+\right. \\
& \left.\Upsilon \otimes\left(h\left(\hat{x}_{k}\right) h^{T}\left(\hat{x}_{k}\right)+C_{k} P_{k} C_{k}^{T}\right)+W_{k}\right]^{-1}
\end{aligned}
$$

The bound on the minimum error covariance equation is

$$
\begin{aligned}
& P_{k+1}=A_{k} P_{k} A_{k}^{T}+V_{k}-\left(A_{k} P_{k} C_{k}^{T} \bar{\Gamma}_{k}^{T}\right)\left[\bar{\Gamma}_{k} C_{k} P_{k} C_{k}^{T} \bar{\Gamma}_{k}^{T}+\right. \\
& \left.\Upsilon \otimes\left(h\left(\hat{x}_{k}\right) h^{T}\left(\hat{x}_{k}\right)+C_{k} P_{k} C_{k}^{T}\right)+W_{k}\right]^{-1}\left(\bar{\Gamma}_{k} C_{k} P_{k} A_{k}^{T}\right)
\end{aligned}
$$

and the state estimate is updated as 


$$
\hat{x}_{k+1}=f\left(\hat{x}_{k}\right)+K_{k}^{o}\left(y_{k}-\bar{\Gamma}_{k} h\left(\hat{x}_{k}\right)\right)
$$

Remark 2

As a more limiting case, if we further neglect the effect of bad data or sensor faults, i.e., $\gamma_{k}^{i}=1$ for all sensors at all time, then $\bar{\Gamma}_{k}$ becomes identity matrix and $\Upsilon$ becomes zero matrix. Clearly, the extended Kalman filter is obtained as a special case of the proposed fault tolerant extended Kalman filter.

\section{SECTION VI.}

\section{Computer Simulation Results}

In Section VI, we compare the performance of the extended Kalman filter (EKF) and the fault tolerant extended Kalman filter (FTEKF) with computer simulations. The average root-mean-square deviation comparison is provided. The sampling frequency is chosen as $2400 \mathrm{~Hz} .[1,1.2,0.8]^{T}$, and $[0, \pi / 3,-2 \pi /$ $3]^{T}$ are chosen as the initial amplitudes, and initial phase angles of the unbalanced three phase voltages, respectively. The additive white Gaussian noise (AWGN) with zero mean and unity matrix covariance is used for simulating measurement noise.

Figs. 1 and 2 show the noisy bad data corrupted measurements. In Fig. 1, the probability of bad data reception in measurement signal $y_{1}$ is $50 \%$. In Fig. 2 , the probability of bad data reception in measure signal $y_{2}$ is $20 \%$. Therefore, we can calculate

$$
\bar{\Gamma}_{k}=\left(\begin{array}{cc}
0.5 & 0 \\
0 & 0.8
\end{array}\right), \Upsilon=\left(\begin{array}{cc}
0.25 & 0 \\
0 & 0.16
\end{array}\right)(48)
$$

Based on these matrices, we can obtain the state estimates using (41), (42), (43). We have performed a number of EKF and FTEKF state estimation experiments applied to grid synchronization. Figs. 3-7 illustrate the fault tolerant extended Kalman filter accurately tracks the five state variables, while EKF fails to converge to the real state trajectories due to the severe nonlinearity cased by bad data. Since EKF uses first order linearization to update the mean of state and the covariance of estimation error, it will fail to converge for high nonlinearity.

In Fig. 8, the performance metric we used to evaluate EKF and FTEKF is the root-mean-square deviation 


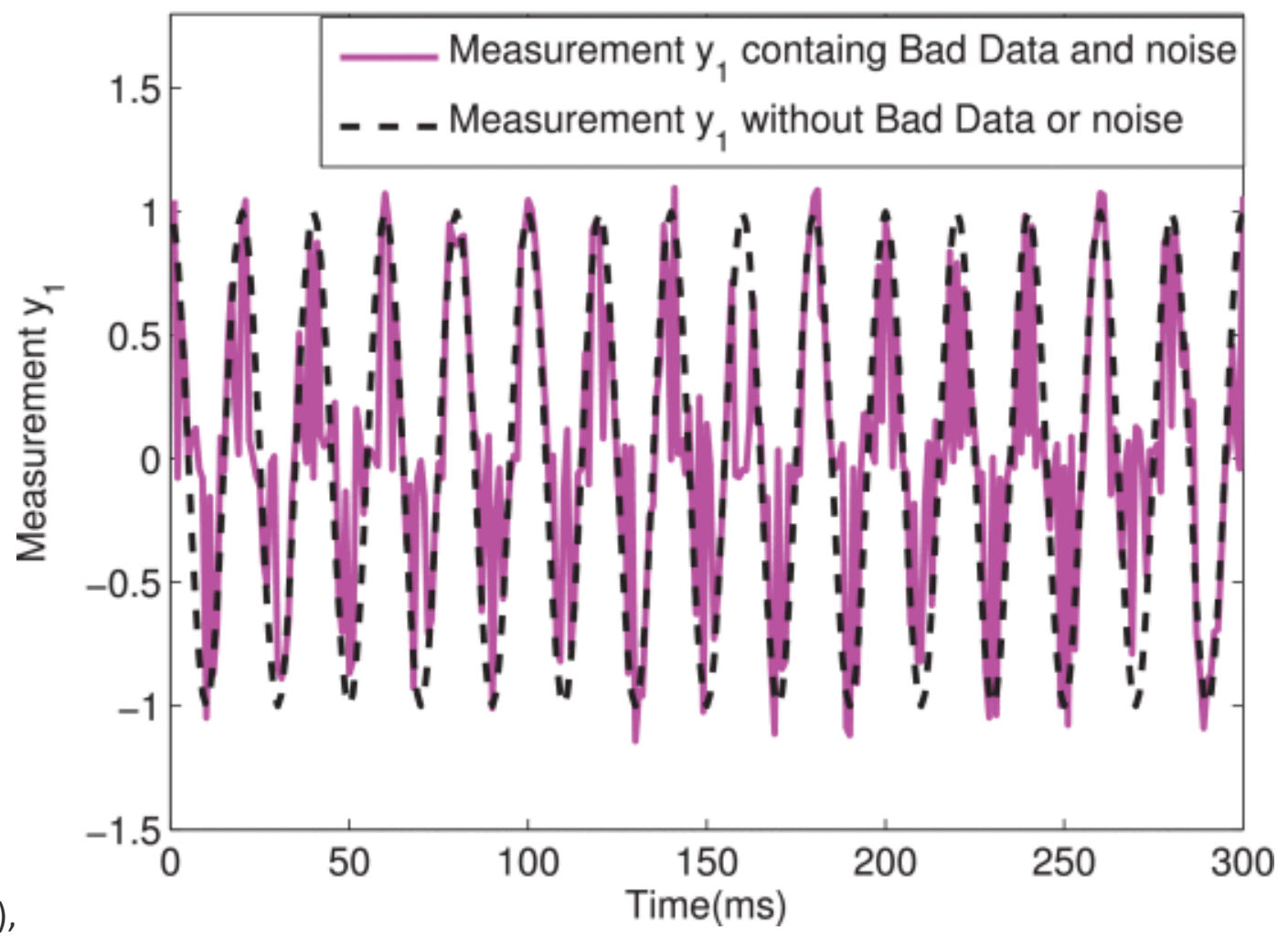

(RMSD),

Fig. 1. Measurement y1.

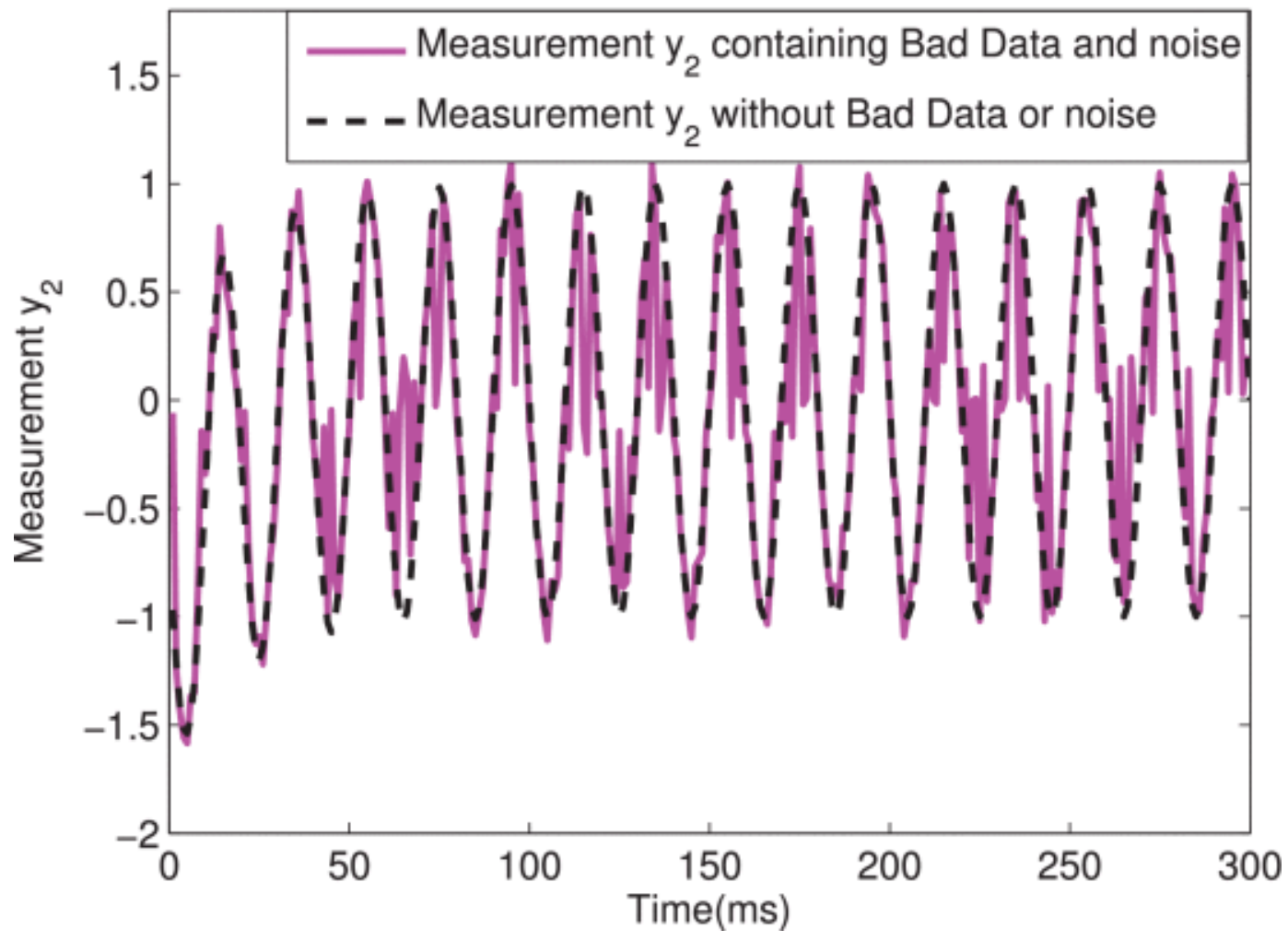

Fig. 2. Measurement y2. 


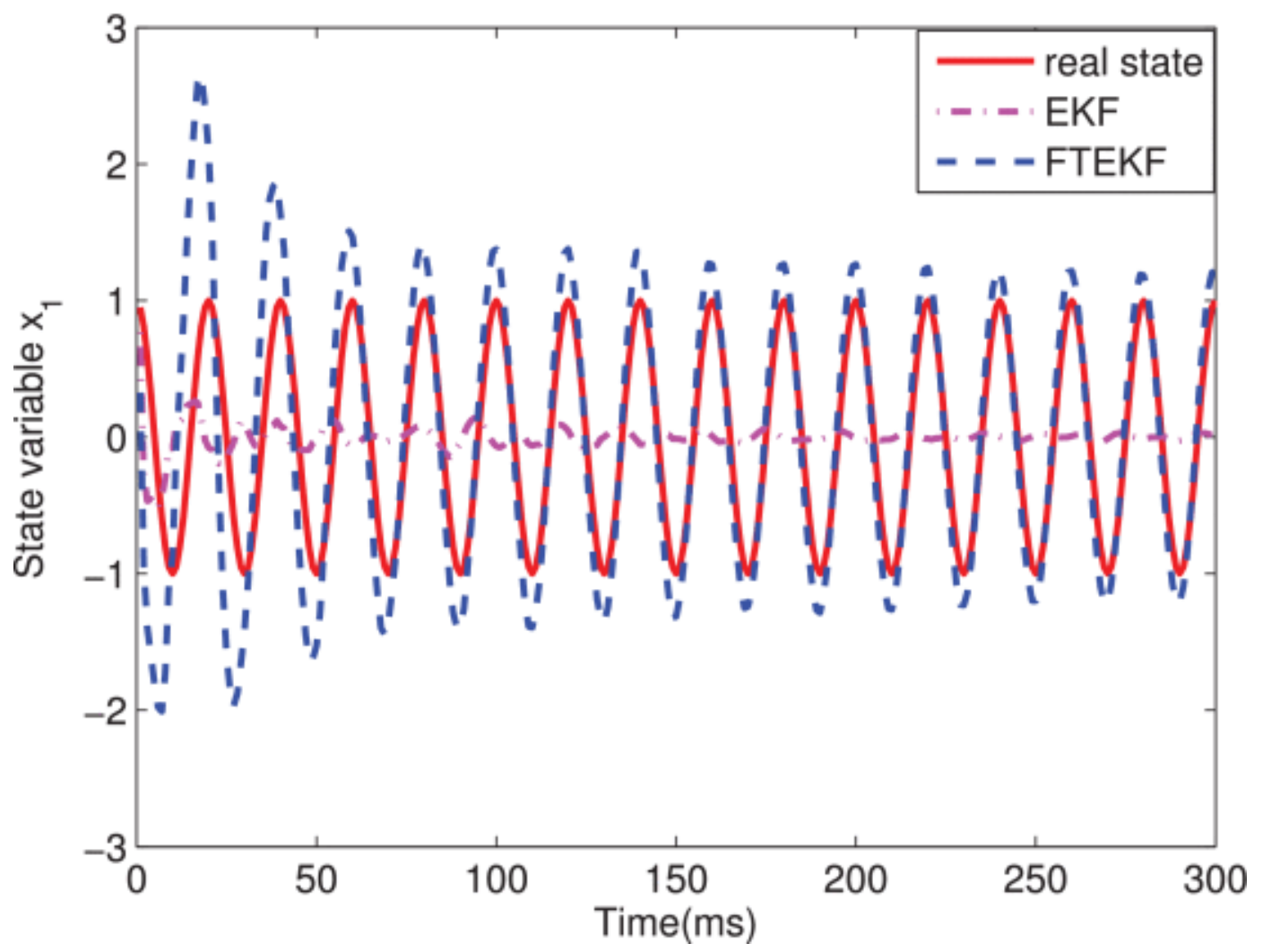

Fig. 3. The first state variable comparison.

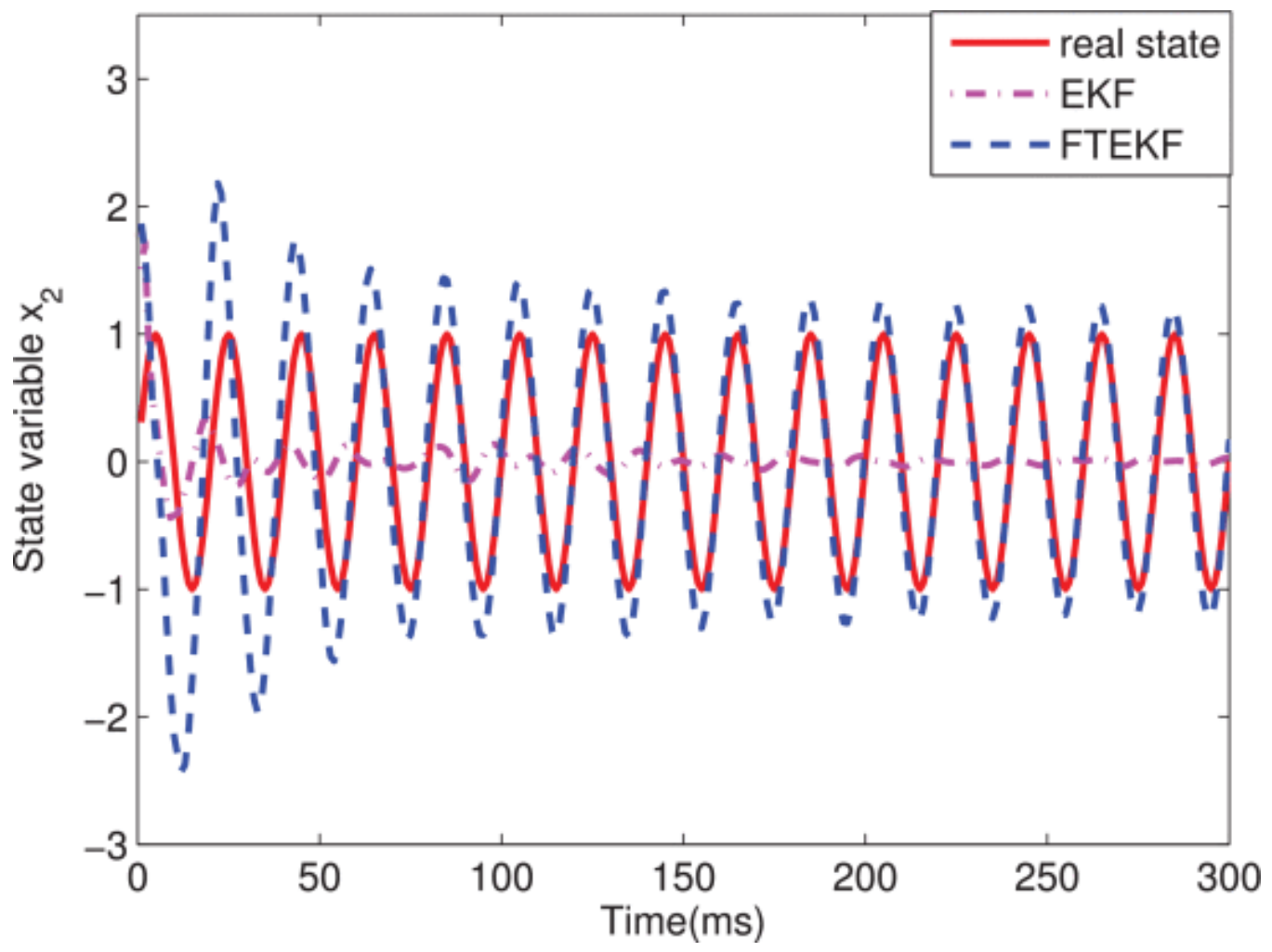

Fig. 4. The second state variable estimation comparison. 


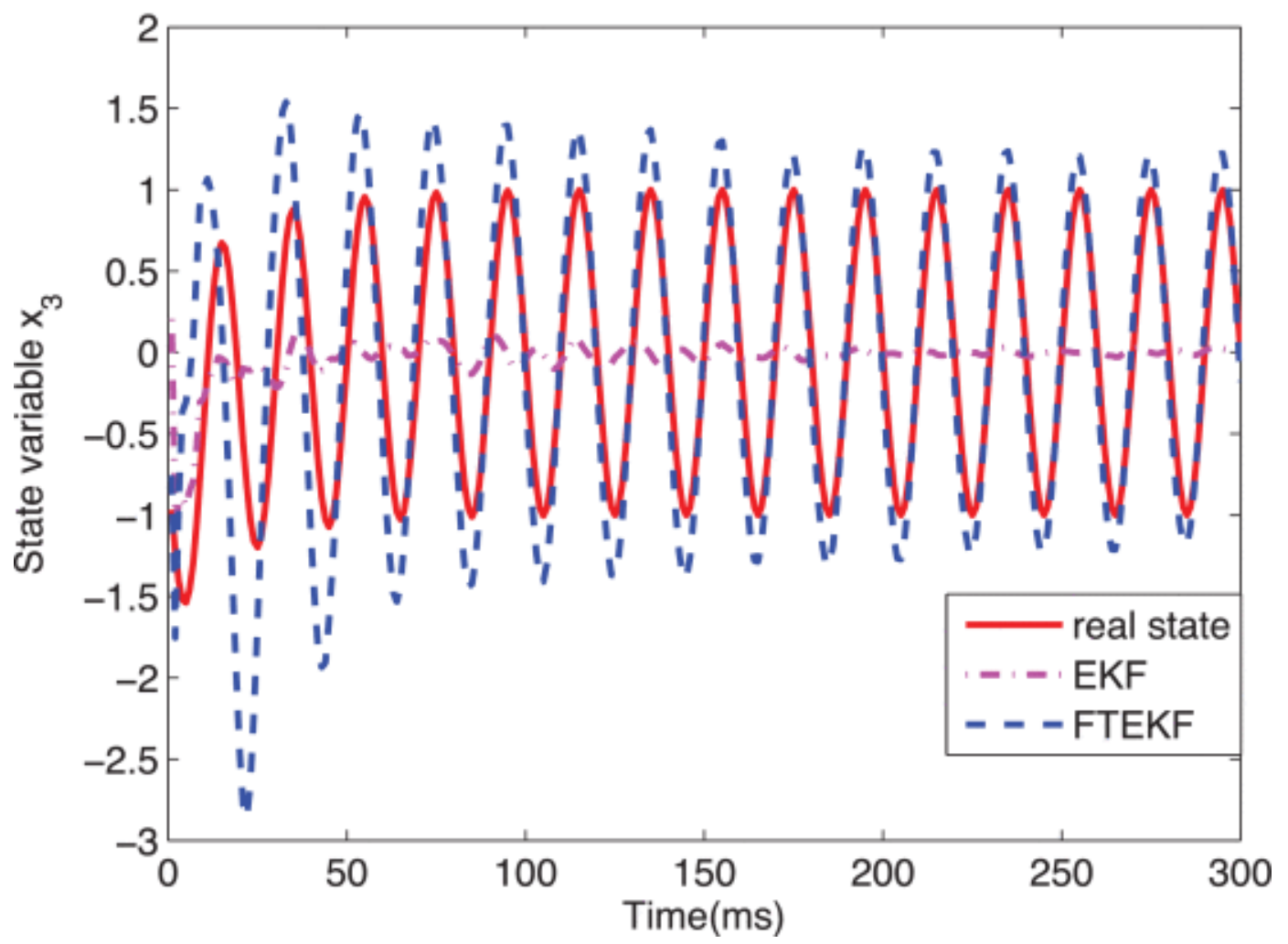

Fig. 5. The third state variable estimation comparison.

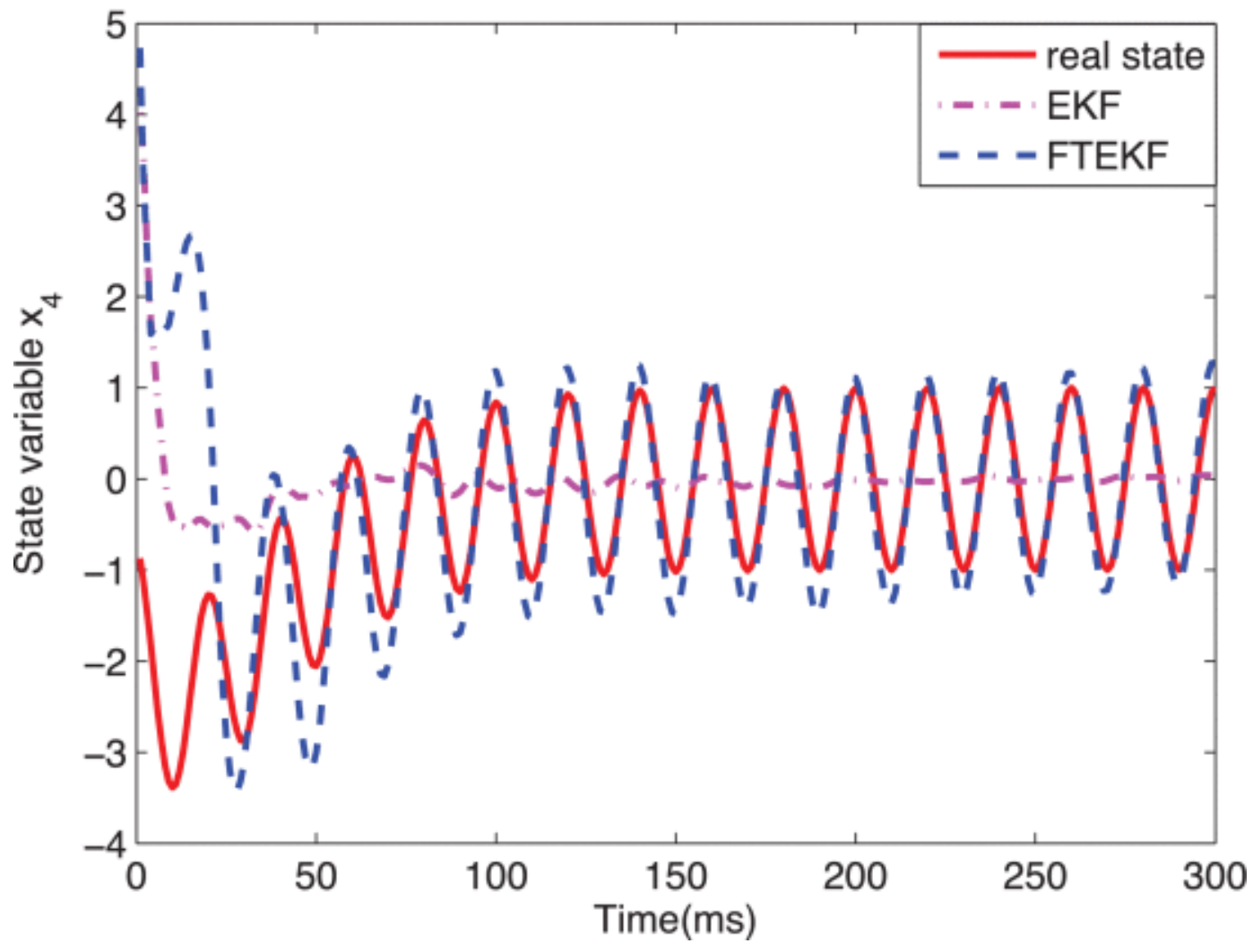

Fig. 6. The fourth state variable estimation comparison. 


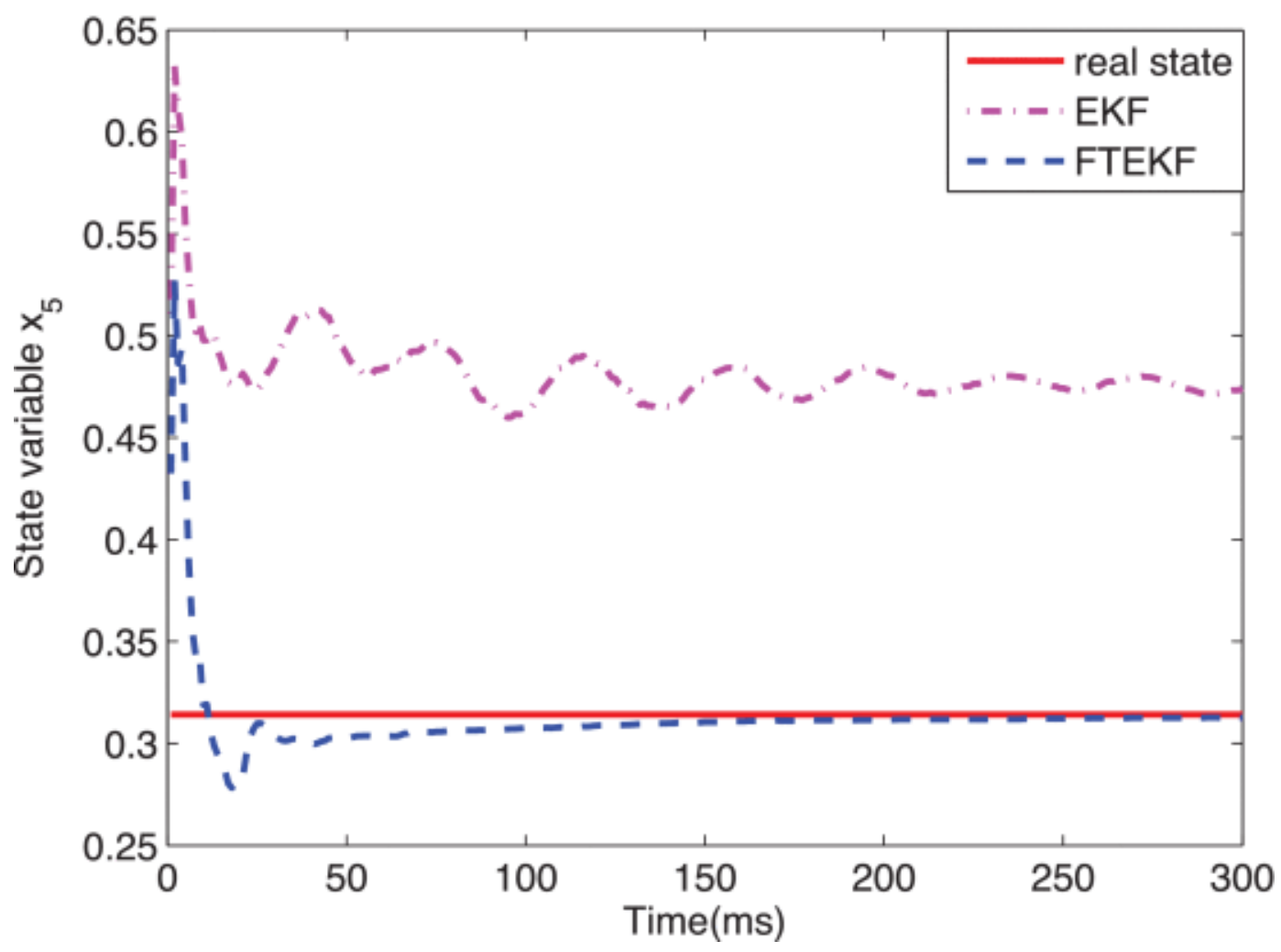

Fig. 7. The fifth state variable estimation comparison.

which is given by

$$
R M S D=\sqrt{\frac{\sum_{k=1}^{n}\left(\hat{x}_{k}-x_{k}\right)^{2}}{n}}
$$

where $\mathrm{n}$ is the number of time steps. 


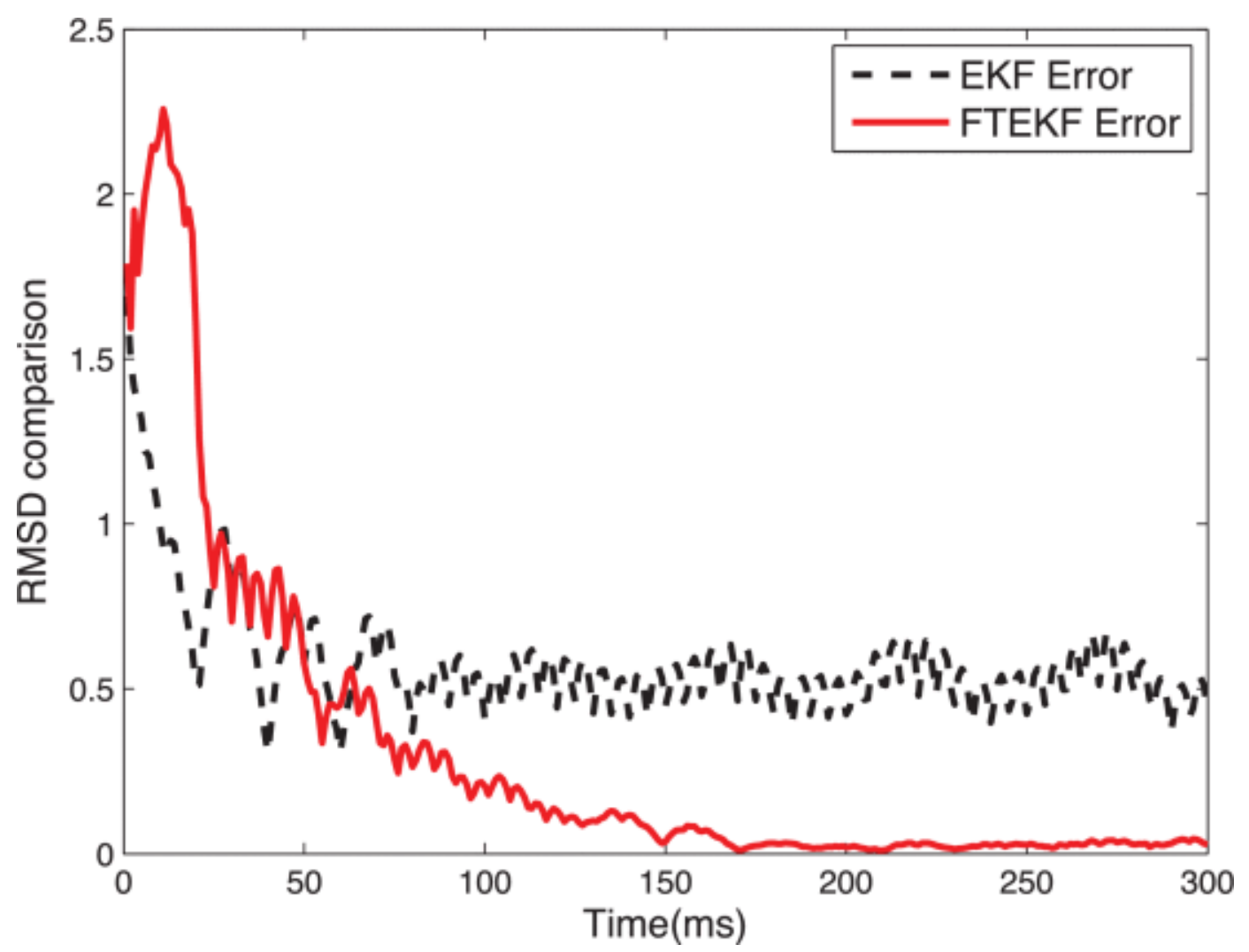

Fig. 8. Average estimation error comparison.

To provide a quantitative view of the performance comparison, Table I shows the RMSD comparison of the two approaches.

Table I RMSD comparison

\begin{tabular}{l||l|l}
\hline Time $(\mathrm{msec})$ & EKF RMSD & FTEKF RMSD \\
\hline 20 & 0.5096 & 1.6125 \\
\hline 50 & 0.5781 & 0.5786 \\
\hline 100 & 0.4056 & 0.2045 \\
\hline 200 & 0.4328 & 0.0287 \\
\hline 300 & 0.4621 & 0.0191 \\
\hline \hline
\end{tabular}

According to the results shown in Table I, at the beginning of the time (from 0 to $50 \mathrm{msec}$ ), EKF shows slightly smaller estimation errors than FTEKF. After the first $50 \mathrm{msec}$, FTEKF effectively tracks the real state trajectories, while EKF fails to converge. By the end of the simulation at $300 \mathrm{msec}$, the average root-meansquare deviation (RMSD) (averaged over 20 experiments with different initial state estimates) for FTEKF is less than 0.0191, comparing with EKF RMSD of 0.4621. Due to the intense nonlinearity caused by bad data, 
EKF is not able to provide reliable state estimates, while FTEKF shows fast convergence speed and small estimation error.

It should also be noted that it takes about 80 ms as shown in Figs. 1 and 3, for FTEKF to track the real state signal accurately. Fig. 8 also shows that for the first $50 \mathrm{~ms}$, the FTEKF RMSD values are slightly larger in comparison with EKF. Also, in the first 50 msec from Table I, FTEKF shows slightly more RMSD than EKF. The reason is that the FTEKF estimation covariance (42) is the upper bound of estimation error covariance matrix, which is obtained by applying Rayleigh's inequality. ${ }^{53}$ At the beginning of estimation, the FTEKF estimation error covariance (42) would be larger than the EKF error covariance, since FTEKF covariance matrix takes sensor measurement failures into account. As time increase, both of the state estimates and covariance converge to the real state values precisely through time iterations of FTEKF. Therefore, after a short period of time (about $60 \mathrm{msec}$ ), FTEKF effectively tracks to the real state signal, while EKF fails.

Finally, the positive sequence phase angle estimate from FTEKF is shown in Fig. 9. Note that only the positive sequence phase angle estimate from FTEKF is compared to the real positive sequence phase angle trajectory, since FTEKF can provide phase angle estimate effectively, while EKF estimation fails. Note the discontinuities of the FTEKF estimates (on the red curves in Fig. 9) are due to the bad data effect.

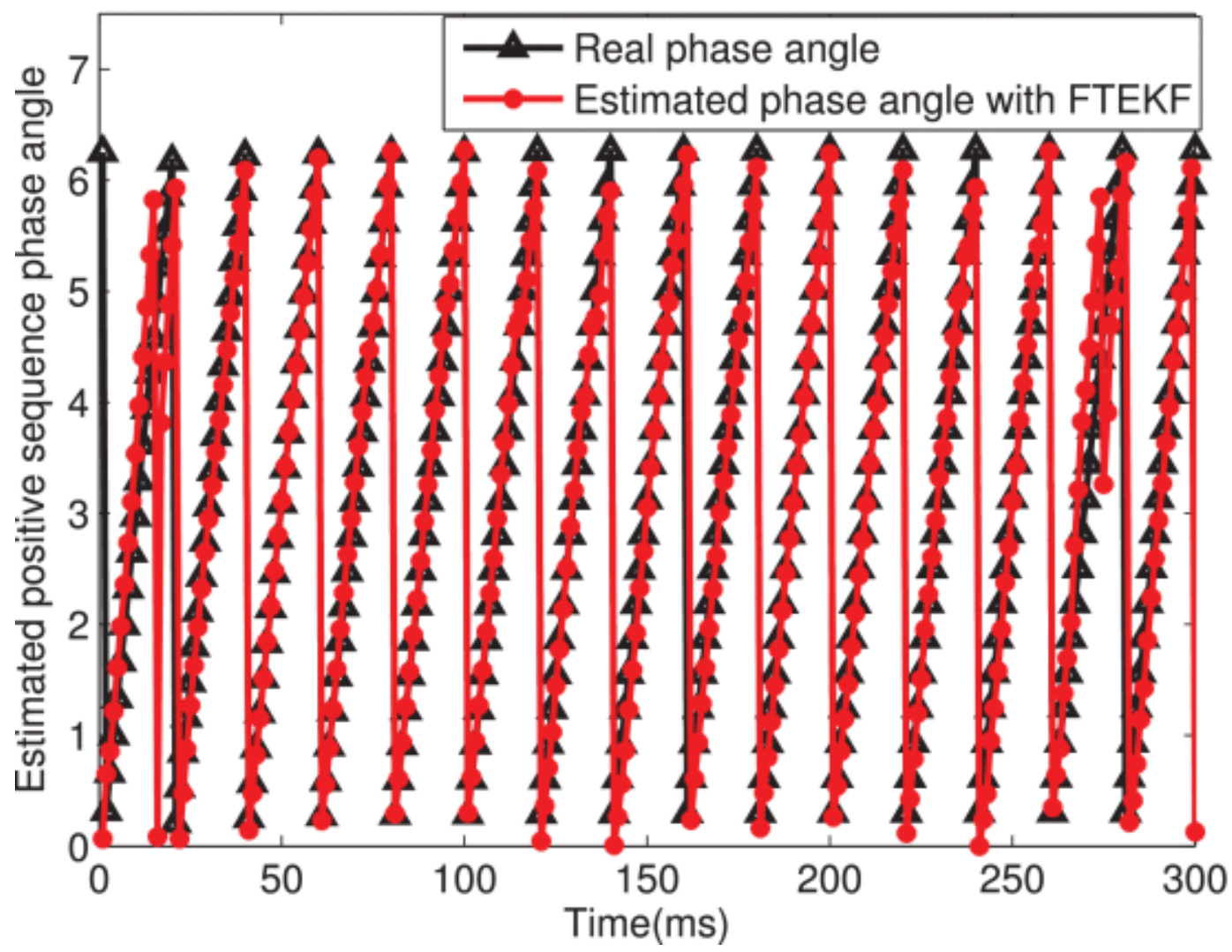

Fig. 9. Positive sequence phase angle estimate with FTEKF. 


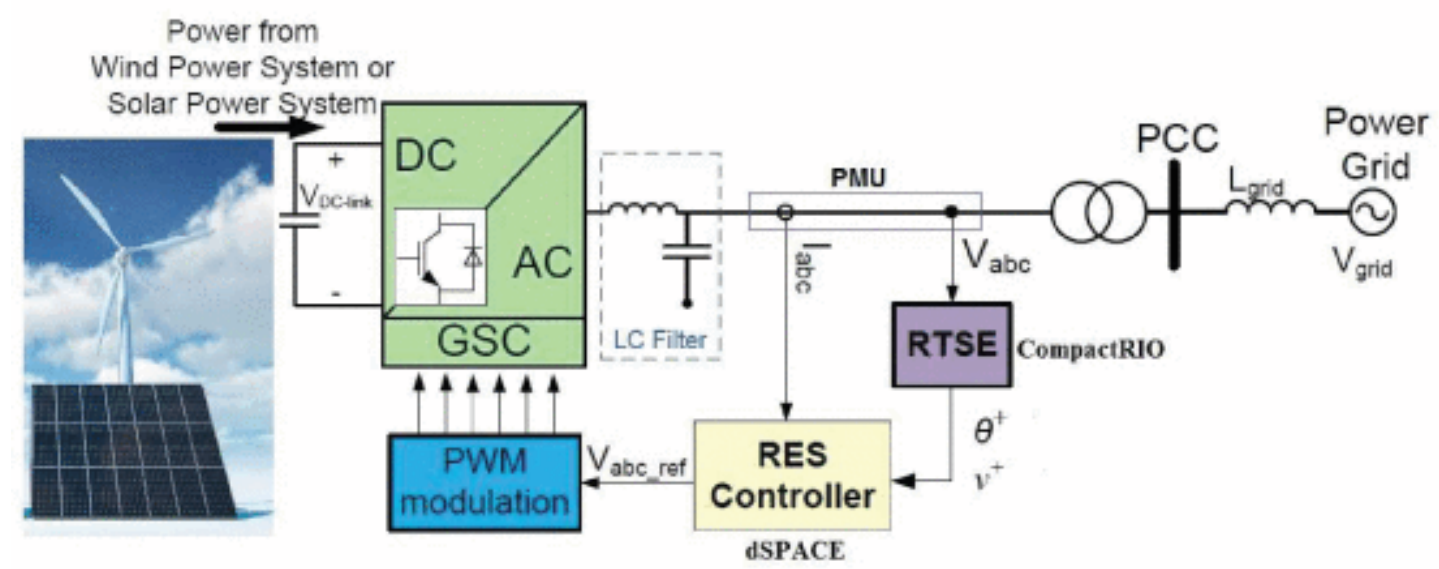

Fig. 10. Structure of An Interconnected Renewable Energy System (RES) with Real Time State Estimator (RTSE). ${ }^{15}$

Results are clearly encouraging based on the averages over experiments, we may conclude the fault tolerant extended Kalman filter shows good estimation accuracy in the presence of bad data, external disturbances and noises.

\section{SECTION VII.}

\section{Hardware Implementation}

The proposed fault tolerant extended Kalman filter (FTEKF) has also been implemented with National Instruments CompactRIO and dSPACE DS1103 hardware platforms Fig. 10. The operation of the interconnected renewable energy sources (RES) is achieved by controlling the grid side converter (GSC), which is ensured by accurate synchronization through real time state estimator (RTSE). ${ }^{15}$ The renewable energy system (RES) controller is implemented with dSPACE DS1103 platform, with PowerPC 750GX microprocessor runs at $1 \mathrm{GHz}$. dSPACE based RES controller can dynamically control the switching of IGBT converters to connect or disconnect distributed generation systems (DG) from or to the power grid. Moreover, real-time state estimator (RTSE) fault tolerant extended Kalman filter algorithm is implemented on the CompactRIO cRIO-9024 platform. The FTEKF RTSE is based on the detection of the phase angle and amplitude of the positive sequence grid voltage (17), (18) at the point of common coupling (PCC). Real-time CompactRIO computes and sends the positive sequence information to dSPACE RES controller.

As shown in Fig. 11, NI 9225 simultaneous analog input module serves as the interface between CompactRIO real time controller and external three phase voltages. The incoming three-phase voltages are connected to three input channels of NI 9225, and the outputs of NI 9225 are fed to the CompactRIO system. In Fig. 12, the three phase voltages are acquired and displayed in LabVIEW front panel. Note that, after the step-down power transformer stage, the three phase voltage signals acquired by NI 9225 are $12.694 \angle 0^{\circ}, 12.978 \angle-119.59^{\circ}$, and $13.078 \angle 120.41^{\circ} \circ$ all at $60.00 \mathrm{~Hz}$. The RMS voltage comparison is also shown in Fig. 12. 


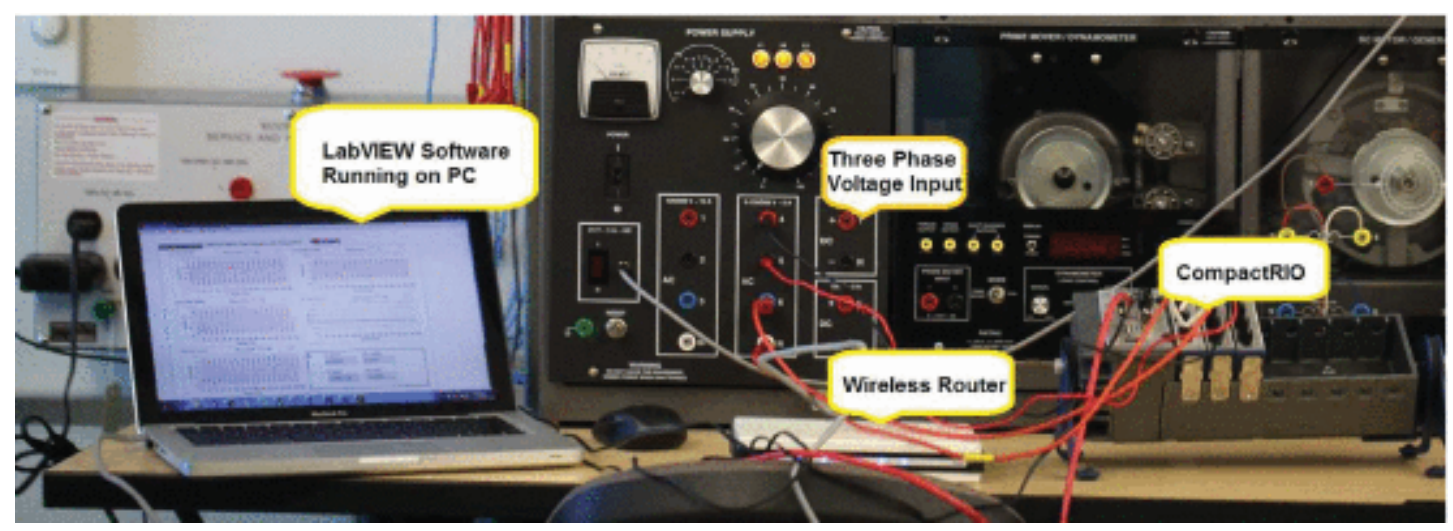

Fig. 11. RTSE hardware implementation.

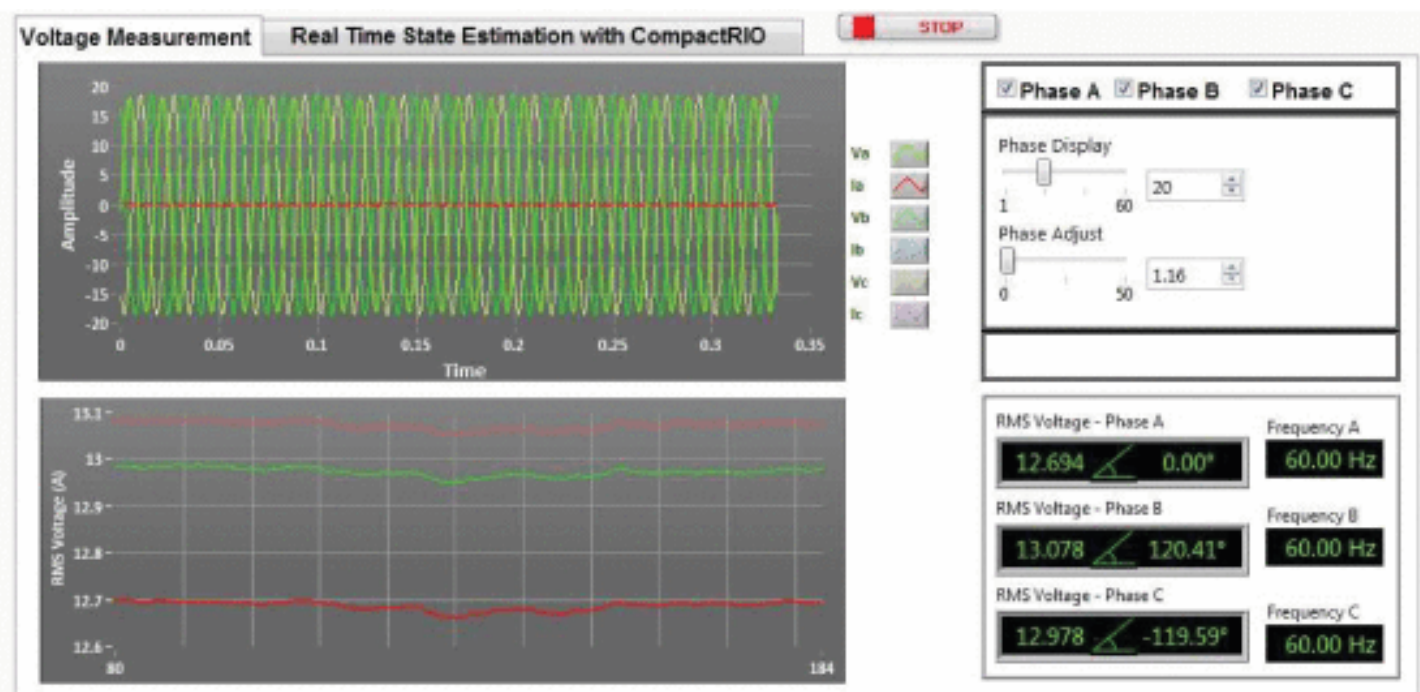

Fig. 12. Real time voltage measurement.

Based on our proposed approach, the estimated state trajectories with FTEKF (shown in blue curves) effectively track the state trajectories (shown in red curves) in Fig. 13 in real time. Hardware-in-the-loop CompactRIO system indicates that the positive sequence voltage $\bar{V}_{p}=12.8085 \angle 13.034^{\circ}$, while the negative sequence component is insignificant. Based on the positive sequence voltage information, dSPACE system can dynamically control the switching of IGBT converters to connect or disconnect distributed generation systems (DG) from or to the power grid. This real-time hardware platform has demonstrated the efficacy of the proposed approach. 


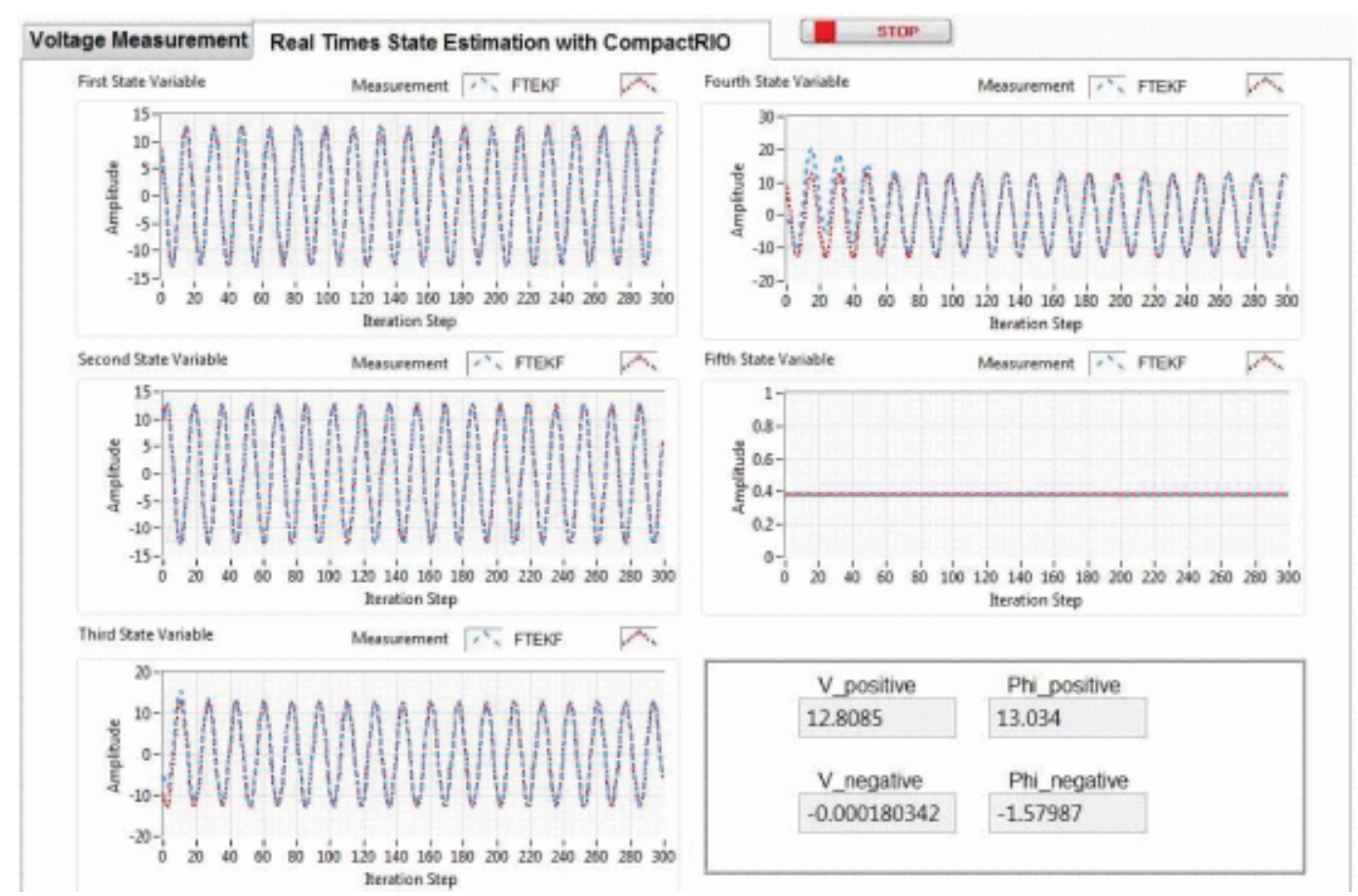

Fig. 13. Real time state estimation with fault tolerant extended Kalman filter.

\section{SECTION VIII.}

\section{Conclusion}

Accurate voltage synchronization under bad data, fault and distorted voltages conditions is a critical concern for properly controlling electrical energy transfer between a distributed power generation system (DPGS) and the grid. This paper have presented a novel fault tolerant extended Kalman filter (FTEKF) with the applications in smart power grid synchronization. We have derived the state space system and measurement equations based on Clarke's transformation and symmetrical component transformation. The extended Kalman filter and the fault tolerant extended Kalman filter have been employed to track the frequency, voltage amplitudes and phase angles for unbalanced three phase voltages with noisy bad data measurements. With the state estimates, the positive sequence phase angle and voltage magnitude can be obtained, which can be used in the energy management systems (EMS) to turn on/off the distributed sources in active distribution networks (ADNs), and therefore to control the active and reactive power flow, and to achieve other specific operation objectives between DGs and the grid. The computer simulation results indicate that FTEKF provides better accuracy than EKF, with similar computational complexity and running time. The proposed FTEKF approach has also been implemented with the dSPACE DS1103 and National Instruments CompactRIO hardware platforms to provide real-time grid synchronization. The impact on the accuracy improvement of the FTEKF RTSE over EKF has been shown to be major, in the view of similar computational complexity. The proposed fault tolerant extended Kalman filter is suitable for RTSE applications in the smart power grid synchronization applications. 


\section{ACKNOWLEDGEMENT}

The authors would like to thank the Editor-in-Chief, Professor Prof. Antonio Conejo, the Editors, and the anonymous reviewers for their insightful comments and suggestions, which greatly helped in improving the quality of this paper.

\section{Definitions}

$f, h$

differentiable non-linear vector functions

$v_{k} \in$ mathcalR $^{n}$

system noise

$w_{k}^{i} \in$ mathcalR

measurement noise in each phasor measurement unit and $w_{k}=\left[w_{k}^{1}, w_{k}^{2}, \ldots, w_{k}^{p}\right]^{T}$

$x_{k} \in$ mathcalR ${ }^{n}$

state vector

$y_{k} \in$ mathcalR

measurement vector

\section{References}

${ }^{1} \mathrm{M}$. H. Bollen, F. Hassan, Integration of Distributed Generation in the Power System, USA, NY, New York:Wiley-IEEE Press, 2011.

${ }^{2}$ F. Katiraei, M. R. Iravani, "Power management strategies for a micro-grid with multiple distributed generation units", IEEE Trans. Power Syst., vol. 21, no. 4, pp. 1821-1831, Nov. 2006.

${ }^{3}$ A. Timbus, M. Liserre, R. Teodorescu, F. Blaabjerg, "Synchronization methods for three phase distributed power generation systems-An overview and evaluation", Proc. IEEE 36th Power Electronics Specialists Conf., pp. 2474-2481, 2005-Jun.

${ }^{4} \mathrm{~F}$. Blaabjerg, R. Teodorescu, M. Liserre, A. V. Timbus, "Overview of control and grid synchronization for distributed power generation systems", IEEE Trans. Ind. Electron., vol. 53, no. 5, pp. 1398-1409, Oct. 2006.

${ }^{5} \mathrm{~V}$. Kekatos, G. Giannakis, "Distributed robust power system state estimation", IEEE Trans. Power Syst., vol. 28, no. 2, pp. 1617-1626, May 2013.

${ }^{6} \mathrm{~S}$. Valiviita, "Zero-crossing detection of distorted line voltages using 1-b measurements", IEEE Trans. Ind. Electron., vol. 46, no. 5, pp. 917-922, Oct. 1999.

${ }^{7} \mathrm{M}$. Azrik, K. H. Ahmed, S. J. Finney, B. W. Williams, "Voltage synchronization scheme based on zero crossing detection for parallel connected inverters in AC microgrids", Proc. 2012 IEEE Int. Symp. Industrial Electronics, pp. 588-593, 2012-May. 
${ }^{8}$ D. Dong, B. Wen, D. Boroyevich, P. Mattavelli, Y. Xue, "Analysis of phase-locked loop low-frequency stability in three-phase grid-connected power converters considering impedance interactions", IEEE Trans. Ind. Electron., vol. 62, no. 1, pp. 310-321, Jan. 2015.

${ }^{9}$ G. Escobar, M. F. Martinez-Montejano, A. A. Valdez, P. R. Martinez, M. Hernandez-Gomez, "Fixedreference-frame phase-locked loop for grid synchronization under unbalanced operation", IEEE Trans. Ind. Electron., vol. 58, no. 5, pp. 1943-1951, May 2011.

${ }^{10}$ Y. Park, S. K. Sul, W. C. Kim, H. Y. Lee, "Phase-locked loop based on an observer for grid synchronization", IEEE Trans. Ind. Applicat., vol. 50, no. 2, pp. 1256-1265, Mar. 2014.

${ }^{11} \mathrm{G}$. Escobar, S. Pettersson, C. N. Ho, "Phase-locked loop for grid synchronization under unbalanced operation and harmonic distortion", Proc. 37th Annu. Conf. IEEE Industrial Electronics Soc., pp. 675680, 2011-Nov.

${ }^{12}$ S. Lee, J.-H. Lee, H. Cha, "Grid synchronization PLL robust to frequency variation unbalanced and distorted voltage", Prof. 2011 IEEE Energy Conversion Congr. Expo., pp. 1150-1155, 2011-Sep.

${ }^{13}$ Y. Yang, L. Hadjidemetriou, F. Blaabjerg, E. Kyriakides, "Benchmarking of phase locked loop based synchronization techniques for grid-connected inverter systems", Proc. 9th Int. Conf. Power Electronics and ECCE Asia, pp. 2167-2174, 2015-Jun.

${ }^{14}$ L. Hadjidemetriou, E. Kyriakides, F. Blaabjerg, "Synchronization of grid-connected renewable energy sources under highly distorted voltages and unbalanced grid faults", Proc. 39th Annu. Conf. IEEE Industrial Electronics Soc., pp. 1887-1892, 2013-Nov.

${ }^{15} \mathrm{~L}$. Hadjidemetriou, E. Kyriakides, F. Blaabjerg, "An adaptive tuning mechanism for phase-locked loop algorithms for faster time performance of interconnected renewable energy sources", IEEE Trans. Ind. Applicat., vol. 51, no. 2, pp. 1792-1804, Mar. 2015.

${ }^{16}$ F. C. Schweppe, J. Wildes, D. Rom, "Power system static state estimation: Parts I II and III", IEEE Tran. Power App. Syst., vol. PAS-89, no. 1, pp. 120-135, Jan. 1970.

${ }^{17}$ A. S. Debs, R. E. Larson, "A dynamic estimator for tracking the state of a power system", IEEE Trans. Power App. Syst., vol. PAS-89, no. 7, pp. 1670-1678, Sep. 1970.

${ }^{18} \mathrm{~A}$. Abur, A. G. Exposito, Power System State Estimation: Theory and Implementation, USA, NY, New York:Marcel Dekker, 2004.

${ }^{19} \mathrm{~T}$. V. Cutsem, M. Ribbens-Pavella, "Critical survey of hierarchical methods for state estimation of electric power systems", IEEE Trans. Power App. Syst., vol. PAS-102, no. 10, pp. 3415-3424, Oct. 1983.

${ }^{20}$ D. M. Falcao, F. F. Wu, L. Murphy, "Parallel and distributed state estimation", IEEE Trans. Power Syst., vol. 10, no. 2, pp. 724-730, May 1995.

${ }^{21}$ R. Ebrahimian, R. Baldick, "State estimation distributed processing", IEEE Trans. Power Syst., vol. 15, no. 4, pp. 1240-1246, Nov. 2000.

${ }^{22} \mathrm{~A}$. J. Conejo, S. de la Torre, M. Canas, "An optimization approach to multi-area state estimation", IEEE Trans. Power Syst., vol. 22, no. 1, pp. 213-231, Feb. 2007.

${ }^{23} \mathrm{~F}$. Pasqualetti, R. Carli, F. Bullo, "Distributed estimation via iterative projections with application to power network monitoring", Automatica, vol. 48, no. 5, pp. 747-758, 2012.

${ }^{24}$ L. Zhao, A. Abur, "Multi-area state estimation using synchronized phasor measurements", IEEE Trans. Power Syst., vol. 20, no. 2, pp. 611-617, May 2005.

${ }^{25} \mathrm{M}$. Zhou, V. A. Centeno, J. S. Thorp, A. G. Phadke, "An alternative for including phasor measurements in state estimators", IEEE Trans. Power Syst., vol. 21, no. 4, pp. 1930-1937, Nov. 2006. 
${ }^{26}$ W. Jiang, V. Vittal, G. T. Heydt, "A distributed state estimator utilizing synchronized phasor measurements", IEEE Trans. Power Syst., vol. 22, no. 2, pp. 563-571, May 2007.

${ }^{27}$ G. N. Korres, "A distributed multi-area state estimation", IEEE Trans. Power Syst., vol. 26, no. 1, pp. 73-84, Feb. 2011.

${ }^{28}$ R. F. Nuqui, A. G. Phadke, "Hybrid linear state estimation utilizing synchronized phasor measurements", Proc. IEEE Power Tech., pp. 1665-1669, 2007-Jul.

${ }^{29}$ N. M. Manousakis, G. N. Korres, J. N. Aliprantis, G. P. Vavourakis, G. C. J. Makrinas, "A two-stage state estimator for power systems with PMU and SCADA measurements", Proc. 2013 IEEE PowerTech Conf., pp. 1-6, 2013-Jun.

${ }^{30}$ S. Chakrabarti, E. Kyriakides, G. Valverde, V. Terzija, "State estimation including synchronized measurements", Proc. 2009 IEEE PowerTech Conf., pp. 1-5, 2009-Jun.

${ }^{31}$ S. Chakrabarti, E. Kyriakides, "PMU measurement uncertainty considerations in WLS state estimation", IEEE Trans. Power Syst., vol. 24, no. 2, pp. 1062-1071, May 2009.

32J. Zhu, A. Abur, "Effect of phasor measurements on the choice of reference bus for state estimation", Proc. IEEE Power Eng. Soc. General Meeting, pp. 1-5, 2007-Jun.

${ }^{33} \mathrm{G}$. N. Korres, N. M. Manousakis, "State estimation and bad data processing for systems including PMU and SCADA measurements", Elect. Power Syst. Res., vol. 81, no. 4, pp. 1514-1524, Jul. 2011.

34J. Zhu, A. Abur, "Bad data identification when using phasor measurements", Proc. 2007 IEEE PowerTech Conf., pp. 1676-1681, 2007-Jul.

${ }^{35} \mathrm{~A}$. Tarali, A. Abur, "Bad data detection in two-stage state estimation using phasor measurements", Proc. $20123 r d$ IEEE PES Int. Conf. Exhib. Innovative Smart Grid Technologies, pp. 1-8, 2012-Oct.

${ }^{36}$ P. Yang, Z. Tan, A. Wiesel, A. Nehora, "Power system state estimation using PMUs with imperfect synchronization", IEEE Trans. Power Syst., vol. 28, no. 4, pp. 4162-4172, Nov. 2013.

${ }^{37} \mathrm{~S}$. Bolognani, R. Carli, M. Todescato, "State estimation in power distribution networks with poorly synchronized measurements", 2014 IEEE 53rd Annu. Conf. Decision and Control, pp. 2579-2584, 2014-Dec.

38J. Zhao, G. Zhang, K. Das, G. N. Korres, N. M. Manousakis, A. K. Sinha, Z. He, "Power system real-time monitoring by using PMU-based robust state estimation method", IEEE Trans. Smart Grid, vol. 7, no. 1, pp. 300-309, Jan. 2016.

${ }^{39}$ D. M. Falcao, P. A. Cooke, A. Brameller, "Power system tracking state estimation and bad data processing", IEEE Trans. Power App. Syst., vol. PAS-101, no. 2, pp. 325-333, Feb. 1982.

${ }^{40}$ Y. Huang, M. Esmalifalak, H. Nguyen, R. Zheng, Z. Han, H. Li, L. Song, "Bad data injection in smart grid: Attack and defense mechanisms", IEEE Commun. Mag., vol. 51, no. 1, pp. 27-33, Jan. 2013.

${ }^{41} \mathrm{P}$. Kabore, H. Wang, "Design of fault diagnosis filters and fault-tolerant control for a class of nonlinear systems", IEEE Trans. Autom. Control, vol. 46, no. 11, pp. 1805-1810, Nov. 2001.

${ }^{42} \mathrm{M}$. Ilic, F. K. Mak, "A new class of fast nonlinear voltage controllers and their impact on improved transmission capacity", Proc. 1989 Amer. Control Conf., pp. 1246-1251, 1989-Jun.

43J. Zhang, A. K. Swain, S. K. Nguang, "Robust sensor fault estimation and fault-tolerant control for uncertain Lipschitz nonlinear systems", Proc. 2014 Amer. Control Conf., pp. 5515-5520, 2014-Jun.

${ }^{44}$ A. von Meier, D. Culler, A. McEachern, "Micro-synchrophasors for distribution systems", Proc. 2013 IEEE Innovative Smart Grid Technologies Conf., pp. 1-6, 2013. 
45J. D. L. Ree, V. Centeno, J. S. Thorp, A. G. Phadke, "Synchronized phasor measurement applications in power systems", IEEE Trans. Smart Grid, vol. 1, no. 1, pp. 20-27, Jun. 2010.

${ }^{46}$ R. O. Burnett, M. M. Butts, T. W. Cease, V. Centeno, G. Michel, R. J. Murphy, A. G. Phadke, "Synchronized phasor measurements of a power system event", IEEE Trans. Power Syst., vol. 9, no. 3, pp. 16431650, Aug. 1994.

${ }^{47}$ I. Dzafic, H. T. Neisius, M. Gilles, S. Henselmeyer, V. Landerberger, "Three-phase power flow in distribution networks using Fortescue transformation", IEEE Trans. Power Syst., vol. 28, no. 2, pp. 1027-1034, May 2013.

48. Dzafic, T. Donlagic, S. Henselmeyer, "Fortescue transformations for three-phase power flow analysis in distribution networks", Proc. 2012 IEEE Power and Energy Soc. General Meeting, pp. 1-7, 2012-Jul.

${ }^{49} \mathrm{M}$. Sun, Z. Sahinoglu, "Extended Kalman filter based grid synchronization in the presence of voltage unbalance for smart grid", Proc. 2011 IEEE PES Innovative Smart Grid Technologies, pp. 1-4, 2011Jan.

${ }^{50} \mathrm{H}$. Darvish, X. Wang, "Synchronization of unbalanced three phase voltages with nonlinear estimation", Proc. 2015 IEEE PES Conf. Innovative Smart Grid Technologies, pp. 1-6, 2015-Feb.

${ }^{51} \mathrm{~K}$. Reif, S. Gunther, E. Yaz, R. Unbehauen, "Stochastic stability of the discrete-time extended Kalman filter", IEEE Trans. Autom. Control, vol. 44, no. 4, pp. 714-728, Apr. 1999.

${ }^{52} \mathrm{~K}$. Reif, S. Gunther, E. Yaz, R. Unbehauen, "Stochastic stability of the continuous-time extended Kalman filter", IEE Proc. - Control Theory Applicat., vol. 147, no. 1, pp. 45-52, Jan. 2000.

${ }^{53} \mathrm{X}$. Wang, E. E. Yaz, "Stochastically resilient extended Kalman filtering for discrete-time nonlinear systems with sensor failures", Int. J. Syst. Sci., vol. 1, no. 9, 2014. 